\title{
Near infrared polarimetry of a sample of YSOs ${ }^{\star}$
}

\author{
A. Pereyra ${ }^{1,2}$, J. M. Girart ${ }^{3}$, A. M. Magalhães ${ }^{2}$, C. V. Rodrigues ${ }^{4}$, and F. X. de Araújo ${ }^{1}$ \\ 1 Observatório Nacional, Rua General José Cristino 77, São Cristovão, 20921-400, Rio de Janeiro, Brazil \\ e-mail: pereyra@on.br \\ 2 Departamento de Astronomia, IAG, Universidade de São Paulo, Rua do Matão 1226, São Paulo, SP, 05508-900, Brazil \\ 3 Institut de Ciències de l'Espai (CSIC- IEEC), Campus UAB - Facultat de Ciències, Torre C5 - parell 2, 08193 Bellaterra, \\ Catalunya, Spain \\ 4 Instituto Nacional de Pesquisas Espaciais/MCT, Avenida dos Astronautas 1758, São José dos Campos, SP, 12227-010, Brazil \\ Received 28 February 2008 / Accepted 24 March 2009
}

\section{ABSTRACT}

\begin{abstract}
Aims. Our goal is to study the physical properties of the circumstellar environment of young stellar objetcs (YSOs). In particular, the determination of the scattering mechanism can help us to constrain the optical depth of the disk and/or envelope in the near infrared. Methods. We used the IAGPOL imaging polarimeter along with the CamIV infrared camera at the LNA observatory to obtain near infrared polarimetry measurements in the $H$ band of a sample of optically visible YSOs, namely, eleven T Tauri stars and eight Herbig Ae/Be stars. An independent determination of the disk (or jet) orientation was obtained for twelve objects from the literature. The circumstellar optical depth could then be estimated by comparing the integrated polarization position angle (PA) with the direction of the major axis of the disk projected onto the plane of the sky. Optically thin disks have, in general, a polarization PA that is perpendicular to the disk plane. In contrast, optically thick disks have polarization PAs parallel to the disks.

Results. Among the T Tauri stars, three are consistent with having optically thin disks (AS 353A, RY Tau and UY Aur) and five with optically thick disks (V536 Aql, DG Tau, DO Tau, HL Tau and LkH $\alpha$ 358). Among the Herbig Ae/Be stars, two stars exhibit evidence of optically thin disks (Hen 3-1191 and VV Ser) and two of optically thick disks (PDS 453 and MWC 297). Our results seem consistent with optically thick disks at near infrared bands, which are more likely to be associated with younger YSOs. Marginal evidence of polarization reversal is found in RY Tau, RY Ori, WW Vul, and UY Aur. In the first three cases, this feature can be associated with the UXOR phenomenon. Correlations with the IRAS colors and the spectral index yielded evidence of an evolutionary segregation in which the disks tend to be optically thin when they are older.
\end{abstract}

Key words. polarization - infrared: stars - stars: pre-main sequence - circumstellar matter

\section{Introduction}

The study of the circumstellar environment in young stellar objects (YSOs) is one of the most interesting topics in star formation and can provide clues about the birth of planetary systems. Polarimetry is a powerful tool for detecting asymmetries in YSOs. This is especially important for non-resolved objects, where the technique provides a direct diagnostic. The scattered emission produced by gas or dust in outflows, jets, and/or disks produces polarization that can give us information about the physical properties of these environments.

Polarimetric observations of YSOs, in general, exhibit temporal variability usually with the polarization position angle (PA) confined to a narrow interval that allows us to infer the presence of a disk (Bastien 1996). Early studies of YSOs comparing the PA of the linear polarization and the direction of the jets, indicated that the jets are aligned preferentially perpendicular (Mundt \& Fried 1983). Single scattering in an optically thin disk (Brown \& McLean 1977) results in a PA perpendicular to the direction of the major axis of the disk projected onto the plane of the sky (hereinafter, the disk PA). On the other hand, optically thick disks may show multiple scattering with the PA parallel to the disk PA (Angel 1969; Bastien \& Ménard 1990). In general, the effect of different disk inclinations alters the polarization level but not the PA in both scenarios. For pole-on objects, no

\footnotetext{
* Based on observations obtained at the Observatório do Pico dos Dias, LNA/MCT, Itajubá, Brazil.
}

net polarization is observed and there is no purpose in defining a PA for these systems.

Alternative models (Whitney \& Hartmann 1992, 1993) predict polarization PA perpendicular to the disk PA in geometrically thin but optically thick disks. These models can also generate polarization PA parallel to the disk PA if there is scattering by material above and below the equatorial disk. In these cases, the optically thick disk requires an additional envelope with bipolar cavities cleared by an outflow from the YSOs.

The statistics from Ménard \& Bastien (1992) is compatible with the prevalent presence of optically thick circumstellar disks surrounding YSOs. Their sample included mainly T Tauri stars (TTS) but also some Herbig Ae/Be stars (HeAeBeS). Maheswar et al. (2002) compiled a sample of HeAeBeS with outflow direction well defined along with polarization measurements and concluded that $55 \%$ of them are consistent with optically thick disks. This seems to indicate that disks in YSOs are usually optically thick. This is also supported by Bastien (1996), who concluded that the polarization observations in YSOs can be explained by multiple scattering by dust grains in a flattened circumstellar disk probably embedded in larger, approximately spherical envelope.

Polarimetry in the near infrared (NIR) is less affected by foreground polarization, something that can be critical in optical bands. Correlations between the NIR polarization PA and any existing asymmetry (such as outflows, jets, or disks) thus allow a better evaluation of the optical depth of the scattering 
mechanism. Compared with the optical, the statistics for NIR polarization measurements of YSOs needs to be improved (Hough et al. 1981; Moneti et al. 1984; Tamura \& Sato 1989; McGregor et al. 1994; Whitney et al. 1997; Perrin et al. 2004; Beckford et al. 2008; Kusakabe et al. 2008). Therefore, a larger NIR sample will help us to clarify whether the trend of optically thick disks at optical wavelengths is also valid in the NIR domain. In addition, the comparison between the optical and NIR polarization PA can provide clues about the detection of a possible polarization PA reversal in YSOs (Hough et al. 1981), caused by the transition from an optically thick disk in the optical to an optically thin environment in the NIR.

Asymmetries in YSOs can be explored using radio, $\mathrm{mm} / \mathrm{submm}$ data, or NIR interferometry. For example, the disk PA can be evaluated using CO emission (Korner \& Sargent 1995) or thermal continuum emission from dust (Kitamura et al. 2002; Qi et al. 2003). NIR interferometry is also useful in detecting the disk PA in YSOs (Akeson et al. 2005; Malbet et al. 2007). On the other hand, the PA of outflows or jets can be obtained from radio extended emission (Anglada 1996). In addition, longslit spectroscopy in forbidden emission lines has also been used to obtain the jet axis in YSOs (Hirth et al. 1994, 1997; Lavalley et al. 1997; Mund \& Eislöffel 1998).

In this work, we combine the detection of asymmetries in YSOs obtained by NIR polarization with the determination of the disk PA from other techniques to constrain the optical properties of the circumstellar disks. Our sample includes selected TTS and HeAeBeS. The observations and data reduction are presented in Sect. 2. The results, including the foreground and intrinsic polarization computation, are presented in Sect. 3. We discuss the correlations with the jet/disk PA and the comparison between optical and NIR polarization PA in Sect. 4. This section also describes the correlations between the disk optical depth, the IRAS colors, and the spectral index. Comments about individual objects are presented in Sect. 5. Our conclusions are drawn in Sect. 6.

\section{Observations}

The observations were completed using IAGPOL, the IAG imaging polarimeter (Magalhães et al. 1996), at the Observatório do Pico dos Dias (OPD), Brazil. Additional details about this instrument can be found in Pereyra (2000) and Pereyra \& Magalhães (2002).

The $0.6 \mathrm{~m}$ and $1.6 \mathrm{~m}$ OPD telescopes were used in several runs between 2003 and 2005. The CamIV infrared camera with an $H$ broadband filter was used along with IAGPOL. CamIV is based on a HAWAII detector (manufactured by Rockwell Sci.) of $1024 \times 1024$ pixels and $18.5 \mu \mathrm{m} /$ pixel that yields a plate scale of $0.5 /$ pixel and $0.25 /$ pixel at the $0.6 \mathrm{~m}$ and $1.6 \mathrm{~m}$ telescopes, respectively. The retarders used by IAGPOL were an achromatic $\lambda / 2$-waveplate centered on $800 \mathrm{~nm}$ (manufactured by Meadowlark Optics, Inc.) and an achromatic $\lambda / 4$-waveplate optimized to NIR (manufactured by B. Halle Nachfl ${ }^{1}$ ). Each object was observed through eight waveplate positions (WP) separated by $22^{\circ} .5$ with five dithered images per WP. A total of $(8 \times 5=) 40$ images were gathered per object. Density filters were used when appropriate.

At each WP, the sky was computed using the mode of the dithered positions. After sky subtraction, flat correction, and registration of the images, the PCCDPACK package (Pereyra 2000) was used to compute the polarimetric parameters. The

${ }^{1}$ http://www.b-halle.de
CamIV's detector yielded a typical area of $8^{\prime}$ and $4^{\prime}$ for the 0.6 and $1.6 \mathrm{~m}$ telescopes, respectively. A log of observations is shown in Table 1 along with optical and NIR magnitudes and extinction estimates for each object.

Observations of unpolarized stars through a Glan prism yielded the effective retardation $(\tau)$, the fiducial zero point of the system (zero), and the polarimetric efficiency of the instrument in each run. The efficiency was always close to $100 \%$ for each ( $\tau$, zero) solution and no efficiency correction was applied to the data. Observations of polarized standard stars in each observing run yielded the position angle correction to the equatorial system $\left(\mathrm{PA}_{\text {corr }}\right)$. Unpolarized standard stars were used to check the instrumental polarization. This was found to be lower than $0.3 \%$; therefore, this correction was not applied to the data. A summary of the calibration data for each run is shown in Table 2.

The selection criteria of our sample was that the YSOs are optically visible and have a measured optical polarization (at least in one epoch) higher than $1 \%$ (see Table 3 ). A summary of the temporal evolution in the optical polarization (mainly at $V$ band) for the objects in our sample is also included in Table 3. The observation epoch for the optical polarization data is also indicated along with the number of measurements per epoch $(N)$, mean values being quoted.

\section{Results}

\subsection{Observed polarization}

The observed polarization for the objects of our sample is shown in Table 4. Column (2) shows the aperture radius (in arcsecs) of the observations. These are computed for a series of apertures around the object; we select the one that minimized the polarization accuracy. This accuracy is calculated from the spread of the measurements at each WP with regards to the 4-cosine curve. Columns (3) and (4) show the observed polarization in $H$ band along with its PA, respectively. As we can note, the NIR polarization level is higher than $1 \%$ for all cases except three T Tauri stars (AS 353A, RY Tau, and UY Aur) and one Herbig Ae/Be (MWC 297). Rather high observed polarization levels (higher than 4\%) are found in three TTS (V536 Aql, HL Tau, and LkH $\alpha$ 358) and in one HeAeBeS (PDS 406).

Only three TTS (RY Tau, DG Tau, and HL Tau) of our sample have a previous NIR polarization measurement in $H$ band (see Table 7). For the remaining objects, we present their first NIR polarization data, to the best of our knowledge.

\subsection{Foreground polarization}

To estimate the NIR foreground polarization toward each target, we searched the literature for similar previous estimates in the optical. Four objects have the optical foreground polarization already computed: AS 353A (Monin et al. 2006), RY Tau (Petrov et al. 1999), MWC 297 (Hillenbrand et al. 1992), and WW Vul (Grinin et al. 1988). Additional optical foreground polarization for seven objects of our sample was obtained from a polarimetric survey of HeAeBeS (Rodrigues et al. 2009). In this survey, the foreground polarization was estimated by the weighted average of the polarization of field stars within typically a $10^{\prime} \times 10^{\prime}$ area around the objects. This foreground estimate was choosen when more than one computation was available (as in MWC 297 and WW Vul).

We assumed that the foreground polarization could be represented by a standard Serkowski law, $P=P_{\max } \exp \left[-K \ln ^{2}\left(\lambda_{\max } / \lambda\right)\right]$ 
Table 1. Log of observations.

\begin{tabular}{|c|c|c|c|c|c|c|c|}
\hline Object & Other name & $V^{a}(\mathrm{mag})$ & $H^{b}$ (mag) & $A_{\mathrm{V}}^{c}(\mathrm{mag})$ & Date & Telescope & Total IT $^{d}(\mathrm{~s})$ \\
\hline \multicolumn{8}{|c|}{ T Tauri } \\
\hline V895 Sco & Haro 1-1 & 13.3 & 9.3 & 1.4 & 2005 Jun. 25 & $1.6 \mathrm{~m}$ & 60 \\
\hline AS 353A & V1352 Aql & 12.5 & 9.2 & 5.7 & 2005 Jun. 25 & $1.6 \mathrm{~m}$ & 60 \\
\hline PX Vul & Hen $3-1751$ & 11.7 & 8.5 & 1.8 & 2005 Jun. 25 & $1.6 \mathrm{~m}$ & 60 \\
\hline V536 Aql & & 12.6 & 8.1 & 1.0 & 2005 Jun. 24 & $1.6 \mathrm{~m}$ & 60 \\
\hline RY Tau & HD 283571 & 10.2 & 6.1 & 0.5 & 2005 Oct. 12 & $0.6 \mathrm{~m}$ & 32 \\
\hline DG Tau & & 12.8 & 7.7 & 3.3 & 2005 Oct. 12 & $0.6 \mathrm{~m}$ & 120 \\
\hline DO Tau & & 13.5 & 8.2 & 4.1 & 2005 Oct. 13 & $0.6 \mathrm{~m}$ & 200 \\
\hline HL Tau & Haro 6-14 & 14.6 & 9.2 & 2.2 & 2005 Oct. 12 & $0.6 \mathrm{~m}$ & 400 \\
\hline $\mathrm{LKH} \alpha 358^{e}$ & & 19.0 & 10.9 & 2.2 & 2005 Oct. 12 & $0.6 \mathrm{~m}$ & 400 \\
\hline UY Aur & & 12.4 & 8.0 & 1.9 & 2005 Oct. 13 & $0.6 \mathrm{~m}$ & 140 \\
\hline RY Ori & Haro 5-82 & 11.8 & 8.9 & 0.6 & 2003 Sep. 20 & $0.6 \mathrm{~m}$ & 600 \\
\hline \multicolumn{8}{|c|}{ Herbig $\mathrm{Ae} / \mathrm{Be}$} \\
\hline PDS 406 & & 13.9 & 10.9 & 0.8 & 2005 Jun. 24 & $1.6 \mathrm{~m}$ & 600 \\
\hline \multirow[t]{2}{*}{ Hen 3-1191 } & & 13.7 & 8.1 & 1.6 & 2005 Jun. 24 & $1.6 \mathrm{~m}$ & 40 \\
\hline & & & & & 2005 Jun. 26 & $1.6 \mathrm{~m}$ & 40 \\
\hline PDS 453 & & 12.9 & 10.0 & 1.1 & 2005 Jun. 24 & $1.6 \mathrm{~m}$ & 120 \\
\hline MWC 297 & PDS 518 & 12.3 & 4.4 & 5.4 & 2005 Oct. 13 & $0.6 \mathrm{~m}$ & $44^{f}$ \\
\hline VV Ser & & 12.2 & 7.4 & 5.7 & 2005 Jun. 26 & $1.6 \mathrm{~m}$ & 32 \\
\hline PDS 520 & & 14.7 & 8.6 & 5.5 & 2005 Jun. 25 & $1.6 \mathrm{~m}$ & 40 \\
\hline WW Vul & HD 344361 & 10.5 & 8.2 & 0.4 & 2005 Jun. 26 & $1.6 \mathrm{~m}$ & 40 \\
\hline VY Mon & & 12.9 & 6.7 & 2.6 & 2005 Oct. 13 & $0.6 \mathrm{~m}$ & 40 \\
\hline
\end{tabular}

${ }^{a}$ From SIMBAD, except for: RY Ori and VV Ser (from NOMAD catalog, Zacharias et al. 2004), and PDS 520 (from Vieira et al. 2003).

${ }^{b}$ 2MASS magnitude at $H$ band.

${ }^{c}$ From Dobashi et al. (2005).

${ }^{d}$ Total integration time at eight waveplate positions, including five ditherings per position.

${ }^{e}$ Object in the same field of HL Tau.

${ }^{f}$ With density filter DN2.6.

Table 2. Calibration summary.

\begin{tabular}{lllllll}
\hline \hline Run & Telescope & Retarder & $\tau\left(^{\circ}\right)$ & Zero $\left(^{\circ}\right)$ & Efficiency $(\%)$ & PA $_{\text {corr }}\left({ }^{\circ}\right)$ \\
\hline 2003 Sep. & $0.6 \mathrm{~m}$ & $\lambda / 2-800 \mathrm{~nm}$ & 141 & 35 & $100.1(0.2)^{a}$ & +138.0 \\
2005 Jun. & $1.6 \mathrm{~m}$ & $\lambda / 4-\mathrm{NIR}$ & 87 & 47 & $99.4(0.4)^{b}$ & +51.2 \\
2005 Oct. & $0.6 \mathrm{~m}$ & $\lambda / 4-\mathrm{NIR}$ & 87 & 47 & $99.4(1.3)$ & +41.9 \\
\hline
\end{tabular}

Errors in parenthesis.

${ }^{a}$ Mean of three measurements.

${ }^{b}$ Mean of two measurements.

(Serkowski et al. 1975), with the maximum polarization, $P_{\max }$, being assumed to occur at $\lambda_{\max }=0.55 \mu \mathrm{m}$ and the parameter $K$ depending on $\lambda_{\max }$ following the relationship of Whittet et al. (1992). Then, we extrapolated the Serkowski relation to NIR wavelengths and obtained the proper foreground polarization in $H$ band $(1.65 \mu \mathrm{m})$. We assumed that the foreground PA is constant with wavelength. The foreground polarization computed in this way is $1 / 3$ of the $P_{\max }$ assumed and is shown in Cols. (5) and (6) of Table 4. Column (7) indicates the number of field objects used to compute the foreground polarization by Rodrigues et al. (2009). In general, a more robust statistic $(N)$ will yield a more precise estimator.

For the nine objects whose foreground correction is available, just two (RY Tau and MWC 297) appear with a foreground polarization level higher than the observed one. Nevertheless, in all the cases, the foreground correction is lower than $1 \%$ and as expected we have a substantial reduction in the foreground at NIR bands compared to the optical polarization. To help the comparison, Fig. 1 shows for each object the Stokes parameters ${ }^{2}$ $Q-U$ diagram, including the NIR observed polarization and the optical and NIR vectors for the foreground polarization (when

${ }^{2} Q=P \cos (2 \times \mathrm{PA})$ and $U=P \sin (2 \times \mathrm{PA})$. available). Analyzing these diagrams, it seems clear that the contribution of the foreground polarization to the observed polarization at NIR bands is significantly reduced.

\subsection{Intrinsic polarization}

For those objects with an estimated foreground polarization (Table 4), we obtained the intrinsic polarization. Since the foreground polarization is an additive component included in the observed polarization, the intrinsic Stokes parameters are as follows:

$Q_{\mathrm{int}}=Q_{\mathrm{obs}}-Q_{\mathrm{for}}$

$U_{\text {int }}=U_{\text {obs }}-U_{\text {for }}$.

The intrinsic polarization $\left(P_{\text {int }}\right)$ and its polarization angle $\left(\mathrm{PA}_{\text {int }}\right)$ are obtained from:

$P_{\text {int }}=\left(Q_{\text {int }}^{2}+U_{\text {int }}^{2}\right)^{1 / 2}$

$\mathrm{PA}_{\text {int }}=\frac{1}{2} \arctan \left(U_{\mathrm{int}} / Q_{\mathrm{int}}\right)$.

The intrinsic polarization estimated for the nine objects (two TTS and seven HeAeBeS) are indicated in Cols. (8) and (9) of 
Table 3. Summary of the temporal evolution in the optical polarization of the targets from the literature.

\begin{tabular}{|c|c|c|c|c|c|c|}
\hline Object & Date/epoch & $N$ & Band & $P^{a}(\%)$ & $\mathrm{PA}^{a}\left({ }^{\circ}\right)$ & Ref. \\
\hline \multicolumn{7}{|c|}{ T Tauri } \\
\hline \multirow{2}{*}{ V895 Sco } & $1978 / 05$ & 1 & $V$ & $3.0(0.4)$ & 165 & 1 \\
\hline & $1978 / 05$ & 1 & $R$ & $2.9(0.2)$ & 173 & 1 \\
\hline \multirow[t]{4}{*}{ AS 353A } & $1976 / 07-1978 / 07$ & 7 & $V$ & $1.4(0.1)$ & 145 & 1 \\
\hline & 1976/10-1978/07 & 6 & $R$ & $1.3(0.02)$ & 144 & 1 \\
\hline & $1986 / 08$ & 1 & $R$ & $1.4(0.2)$ & 139 & 4 \\
\hline & $2000 / 05$ & 1 & $I$ & $1.4(0.04)$ & 125 & 5 \\
\hline PX Vul & 1998/07-1998/10 & 9 & $V$ & $3.9(0.1)$ & 28 & 2 \\
\hline \multirow{3}{*}{ V536 Aql } & 1977/08-1978/07 & 2 & $V$ & $6.6(0.3)$ & 67 & 1 \\
\hline & 1977/08-1978/07 & 2 & $R$ & $5.5(0.5)$ & 65 & 1 \\
\hline & 1985/06-1986/08 & 2 & $R$ & $7.6(0.4)$ & 43 & 4 \\
\hline \multirow[t]{8}{*}{ RY Tau } & $1972 / 01$ & 1 & $V$ & $3.2(0.2)$ & 30 & 9 \\
\hline & $1973 / 01$ & 1 & $V$ & $5.0(0.1)$ & 20 & 9 \\
\hline & $1976 / 09-1978 / 12$ & 16 & $V$ & $2.5(0.2)$ & 21 & 1 \\
\hline & $1979 / 09$ & 1 & $V$ & $3.1(0.2)$ & 18 & 8 \\
\hline & 1980/01 & 3 & $V$ & $3.0(0.04)$ & 18 & 6 \\
\hline & $1980 / 02$ & 1 & $V$ & $2.2(0.1)$ & 39 & 8 \\
\hline & 1998/10-1999/01 & 7 & $V$ & $2.6(0.1)$ & 23 & 2 \\
\hline & $2001 / 12-2003 / 12$ & 4 & $R$ & $2.0(0.3)$ & 13 & 10 \\
\hline \multirow[t]{3}{*}{ DG Tau } & 1976/10-1978/10 & 3 & $V$ & $5.5(0.1)$ & 136 & 1 \\
\hline & 1976/10-1978/10 & 2 & $R$ & $6.0(0.1)$ & 135 & 1 \\
\hline & $1980 / 01$ & 1 & $V$ & $5.1(0.1)$ & 137 & 6 \\
\hline \multirow[t]{3}{*}{ DO Tau } & $1976 / 10$ & 1 & $V$ & $3.1(0.2)$ & 171 & 1 \\
\hline & 1976/10 & 1 & $R$ & $2.9(0.1)$ & 171 & 1 \\
\hline & 1980/01 & 1 & $V$ & $3.8(0.3)$ & 175 & 6 \\
\hline \multirow[t]{5}{*}{ HL Tau } & 1976/10 & 1 & $V$ & $8.3(0.4)$ & 146 & 1 \\
\hline & $1976 / 10$ & 1 & $R$ & $11.2(0.2)$ & 147 & 1 \\
\hline & $1980 / 01$ & 1 & $V$ & $13.5(0.8)$ & 143 & 6 \\
\hline & 1985/01 & 1 & $B$ & $13.7(0.3)$ & 138 & 4 \\
\hline & 1987/01 & 1 & clear & $14.0(0.3)$ & 143 & 11 \\
\hline $\mathrm{LkH} \alpha 358$ & 1987/01 & 1 & clear & $4.1(0.3)$ & 6 & 11 \\
\hline \multirow{3}{*}{ UY Aur } & 1978/12/01 & 1 & $V$ & $3.3(0.2)$ & 127 & 1 \\
\hline & $1979 / 02 / 28$ & 1 & $V$ & $2.3(0.5)$ & 162 & 1 \\
\hline & $1980 / 01 / 05$ & 1 & $V$ & $3.0(0.2)$ & 161 & 6 \\
\hline \multirow[t]{3}{*}{ RY Ori } & $1995 / 01 / 30$ & 1 & $V$ & $6.6(1.9)$ & 165 & 7 \\
\hline & $1995 / 01 / 30$ & 1 & $V$ & $4.3(1.9)$ & 41 & 7 \\
\hline & 1998/10-1999/02 & 7 & $V$ & $2.7(0.1)$ & 72 & 2 \\
\hline \multicolumn{7}{|c|}{ Herbig Ae/Be } \\
\hline PDS 406 & 1999/04/11 & 1 & $V$ & $4.7(0.1)$ & 34 & 3 \\
\hline Hen 3-1191 & 1999/04/11 & 1 & $V$ & $5.9(0.2)$ & 46 & 3 \\
\hline PDS 453 & 1999/04/08 & 1 & $V$ & $3.6(0.1)$ & 49 & 3 \\
\hline \multirow[t]{6}{*}{ MWC 297} & 1973/10 & 1 & $V$ & $1.4(0.2)$ & 95 & 12 \\
\hline & 1975/08 & 1 & V & $1.9(0.1)$ & 98 & 12 \\
\hline & 1976/06 & 1 & V & $1.8(0.1)$ & 95 & 12 \\
\hline & 1985(6) & 1 & $V$ & $2.1(0.2)$ & 91 & 13 \\
\hline & 1998/05 & 1 & $V$ & $1.2(0.2)$ & 95 & 2 \\
\hline & $1999 / 07 / 29$ & 1 & V & $1.8(0.1)$ & 94 & 3 \\
\hline \multirow[t]{2}{*}{ VV Ser } & $1998 / 05-1998 / 10$ & 11 & $V$ & $1.7(0.1)$ & 78 & 2 \\
\hline & $2000 / 06 / 22$ & 1 & $V$ & $1.8(0.1)$ & 78 & 3 \\
\hline PDS 520 & $1999 / 07 / 28$ & 1 & $V$ & $3.5(0.1)$ & 15 & 3 \\
\hline \multirow[t]{4}{*}{ WW Vul } & 1987/08 & 3 & $V$ & $5.3(0.6)$ & 170 & 14 \\
\hline & $1998 / 05$ & 3 & V & $0.6(0.1)$ & 93 & 2 \\
\hline & 1998/07-1998/10 & 8 & $V$ & $0.4(0.1)$ & 146 & 2 \\
\hline & $2000 / 06 / 21$ & 1 & $V$ & $0.9(0.1)$ & 151 & 3 \\
\hline \multirow[t]{2}{*}{ VY Mon } & 1995/01-1995/02 & 7 & $V$ & $8.7(0.7)$ & 6 & 7 \\
\hline & $1998 / 10-1999 / 02$ & 3 & $V$ & $9.8(0.5)$ & 5 & 2 \\
\hline
\end{tabular}

Errors in parenthesis.

${ }^{a}$ Mean values quoted when $N>1$.

References: (1) Bastien (1982), the $\sigma_{1}$ error is quoted for individual observations and $\sigma_{2}$ for mean values; for simplicity $V$ and $R$ bands refer to the filters centered in $5895 \AA$ and $7543 \AA$, respectively; only $V$ measurements for RY Tau and UY Aur are quoted; (2) Oudmaijer et al. (2001), only $V$ measurements are quoted; (3) Rodrigues et al. (2009); (4) Ménard \& Bastien (1992); (5) Monin et al. (2006); (6) Bastien (1985), for simplicity $B$ refers to the filter centered in $4700 \AA$ and $R$ to the filters centered in $7640 \AA$ and $7675 \AA ;$ (7) Yudin \& Evans (1998), only $V$ measurements are quoted; (8) Hough et al. (1981); (9) Breger (1974), only V measurements are quoted; (10) Vink et al. (2005); (11) Gledhill \& Scarrott (1989); (12) Vrba et al. (1979), only $V$ measurements are quoted; (13) Hillenbrand et al. (1992); (14) Grinin et al. (1988), mean polarization on the photometric deep minima is quoted. 


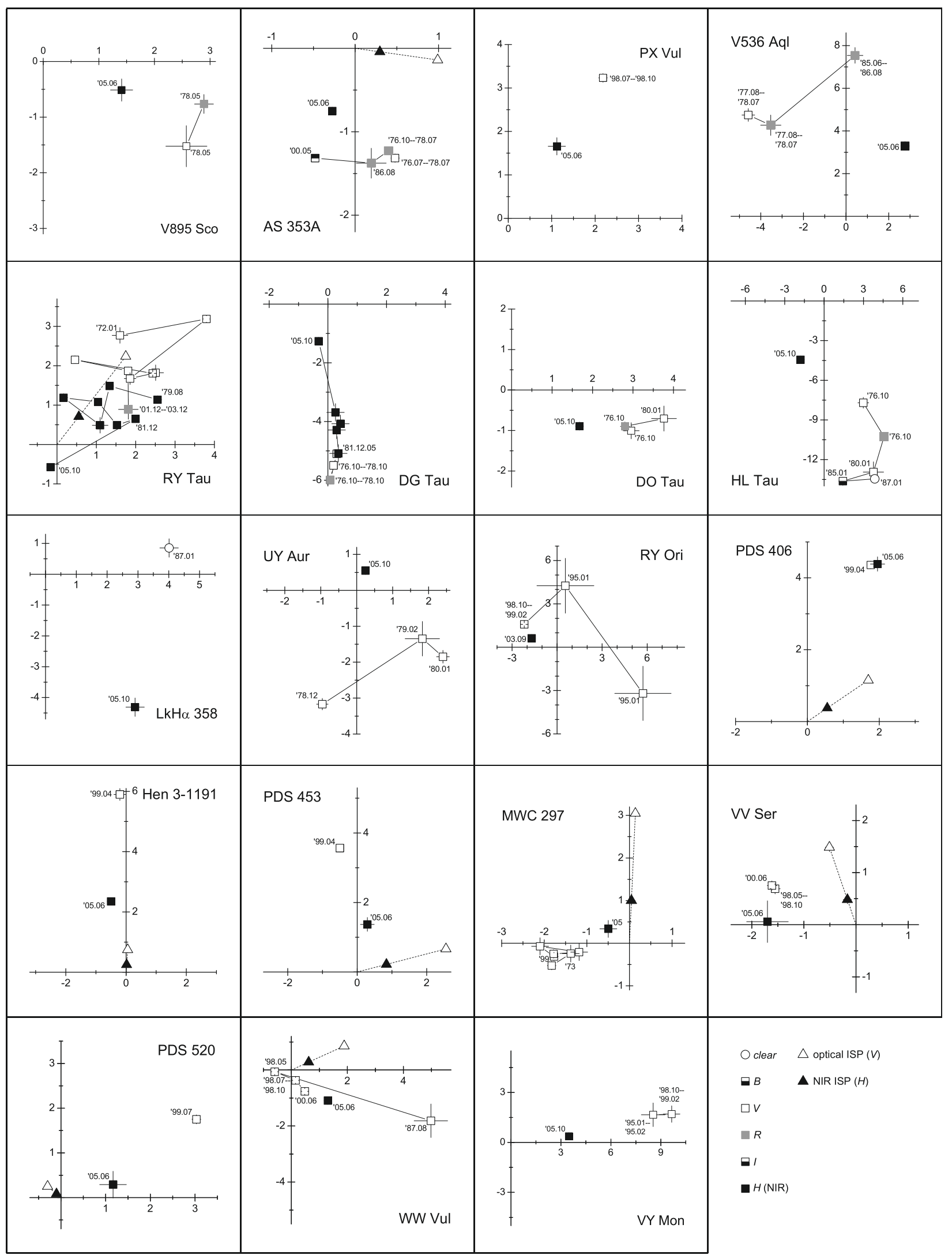

Fig. 1. $Q-U$ diagrams showing the temporal evolution in the objects of our sample. In each plot, the abscissa is the $Q$ parameter, and the ordinate, the $U$ parameter. Units are given in percentages (\%). The optical polarization data refer to Table 3 . The measurements in clear filter are in white circles (only in HL Tau and $\mathrm{LkH} \alpha 358$ ), $V$ in white boxes, $R$ in grey boxes, $I$ in black-white boxes (only in AS 353A), and $B$ in white-black boxes (only in HL Tau). Our NIR polarization data (Table 4) and multiple epoch NIR data of RY Tau and DG Tau (Table 7) are shown in black boxes. The optical (and NIR, when available) temporal evolution is indicated by solid lines and labels. The direction of the foreground polarization, when available, is indicated by a dotted line. White and black triangles represent the optical and NIR foreground polarization vector, respectively. 
Table 4. NIR linear polarization measurements.

\begin{tabular}{|c|c|c|c|c|c|c|c|c|}
\hline \multirow[b]{2}{*}{ Object } & \multicolumn{3}{|c|}{ Observed Pol. } & \multicolumn{3}{|c|}{ Foreground Pol. $^{a}$} & \multicolumn{2}{|c|}{ Intrinsic Pol. } \\
\hline & $\begin{array}{l}\text { Aper. } \\
\left({ }^{\prime \prime}\right) \\
(2)\end{array}$ & $\begin{array}{c}P_{\text {obs }} \\
(\%) \\
(3)\end{array}$ & $\begin{array}{c}\mathrm{PA}_{\mathrm{obs}} \\
\left(^{\circ}\right) \\
(4)\end{array}$ & $\begin{array}{c}P_{\text {for }} \\
(\%) \\
(5)\end{array}$ & $\begin{array}{c}\mathrm{PA}_{\text {for }} \\
\left(^{\circ}\right) \\
(6)\end{array}$ & (7) & $\begin{array}{l}P_{\text {int }} \\
(\%) \\
(8)\end{array}$ & $\begin{array}{c}\mathrm{PA}_{\text {int }} \\
\left(^{\circ}\right) \\
(9)\end{array}$ \\
\hline \multicolumn{9}{|c|}{ T Tauri } \\
\hline V895 Sco & 1.0 & $1.5(0.2)$ & $170(4)$ & - & - & - & - & - \\
\hline AS 353A & 1.3 & $0.8(0.04)$ & $125(1)$ & 0.3 & 176 & & $0.9(0.04)$ & $114(1)$ \\
\hline PX Vul & 5.0 & $2.0(0.2)$ & $28(3)$ & - & - & - & - & - \\
\hline V536 Aql & 1.5 & $4.3(0.2)$ & $25(1)$ & - & - & - & - & - \\
\hline RY Tau & 3.5 & $0.6(0.02)$ & $127(1)$ & 0.9 & 26 & & $1.5(0.02)$ & $120(0.4)$ \\
\hline DG Tau & 2.5 & $1.3(0.1)$ & $128(2)$ & - & - & - & - & - \\
\hline DO Tau & 6.0 & $1.9(0.1)$ & $166(1)$ & - & - & - & - & - \\
\hline HL Tau & 0.5 & $4.8(0.2)$ & $124(1)$ & - & - & - & - & - \\
\hline $\mathrm{LkH} \alpha 358$ & 1.0 & $5.2(0.3)$ & $152(2)$ & - & - & - & - & - \\
\hline UY Aur & 6.0 & $0.6(0.1)$ & $33(5)$ & - & - & - & - & - \\
\hline RY Ori & 5.5 & $1.8(0.1)$ & $80(1)$ & - & - & - & - & - \\
\hline \multicolumn{9}{|c|}{ Herbig Ae/Be } \\
\hline PDS 406 & 1.5 & $4.8(0.2)$ & $33(1)$ & $0.7(0.01)$ & $17(0.4)$ & 348 & $4.2(0.2)$ & $35(1)$ \\
\hline Hen $3-1191^{b}$ & 1.6 & $2.4(0.1)$ & $51(1)$ & $0.2(0.01)$ & $43(1)$ & 546 & $2.1(0.1)$ & $51(1)$ \\
\hline PDS 453 & 1.3 & $1.4(0.2)$ & $39(3)$ & $0.9(0.02)$ & $7(1)$ & 412 & $1.3(0.2)$ & $58(3)$ \\
\hline MWC 297 & 4.0 & $0.6(0.2)$ & $73(10)$ & $1.0(0.09)$ & $44(3)$ & 4 & $0.9(0.2)$ & $114(8)$ \\
\hline VV Ser & 3.5 & $1.7(0.4)$ & $89(6)$ & $0.5(0.1)$ & $55(4)$ & 5 & $1.6(0.4)$ & $98(7)$ \\
\hline PDS 520 & 1.3 & $1.2(0.3)$ & $7(8)$ & $0.1(0.03)$ & $70(6)$ & 9 & $1.2(0.3)$ & $4(8)$ \\
\hline WW Vul & 1.0 & $1.7(0.1)$ & $160(2)$ & $0.7(0.01)$ & $12(0.5)$ & 336 & $1.5(0.1)$ & $148(2)$ \\
\hline VY Mon & 2.5 & $3.5(0.2)$ & $3(2)$ & - & - & - & - & - \\
\hline
\end{tabular}

${ }^{a}$ From Rodrigues et al. (2009) except AS 353A, from Monin et al. (2006), and RY Tau, from Petrov et al. (1999).

${ }^{b}$ Observed polarization is the average of two different nights (2005 Jun. 24 and 2005 Jun. 26).

Table 4. In all cases, the intrinsic polarization levels are significant (higher than $0.9 \%$ ). In seven objects, the difference between the observed and intrinsic polarization levels is less or equal to $0.3 \%$. Only RY Tau and PDS 406 show larger polarization changes after the foreground correction $(0.9 \%$ and $0.6 \%$, respectively). In addition, seven YSOs show small changes in the PA after correcting for foreground polarization, typically similar or less than $10^{\circ}$. Significant deviations are found in PDS $453\left(19^{\circ}\right)$ and MWC $297\left(41^{\circ}\right)$.

As we can note, in general, the subtraction of the foreground polarization does not change the results significantly. For example, the extreme $P_{\text {int }}$ measured for PDS 406 (4.2\%) is comparable with its $P_{\text {obs }}(4.8 \%)$ value with the PA practically unchanged. We can then confirm the small contribution of the foreground polarization. This is important if the foreground polarization is not available, and only $\mathrm{PA}_{\mathrm{obs}}$ can be used to infer correlations with the position angle for the jets/disks.

\section{Discussion}

\subsection{Correlations with position angles of jets and disks}

The main focus of this work is to identify any correlation of the polarization PA with the directions of jets, outflows, or disks/envelope for the objects of our sample available in the literature. We found non-polarimetric evidence of asymmetries in twelve objects of our sample (eight TTS and four HeAeBeS). The PA of disks or jets $\left(\mathrm{PA}_{\text {jet }}\right.$ and $\left.\mathrm{PA}_{\text {disk }}\right)$ are indicated in Table 5 with their respective references.
In five cases, there are independent determinations of $\mathrm{PA}_{\text {jet }}$ and $\mathrm{PA}_{\text {disk }}$, which, within the uncertainties, are perpendicular. This is quantified by the parameter $\Delta_{\text {jet/disk }}$ in Table 5 . For RY Tau, DG Tau, and DO Tau, the angles $\mathrm{PA}_{\text {jet }}$ and $\mathrm{PA}_{\text {disk }}$ are perpendicular to within $4^{\circ}$ and for HL Tau and MWC 297 to within $26^{\circ}$.

The angle difference between the polarization PA and the disk PA is defined as $\Delta_{\text {pol/disk }}$ in Table 5. For an optically thick disk, this difference is $0^{\circ}$, whereas for an optically thin disk the difference is $90^{\circ}$. We classified the disk optical depth preferentially using the intrinsic polarization PA. If it was unavailable, the observed value was used.

In the twelve objects for which the comparison was completed, the jet/disk PA is at least within $14^{\circ}$ of being parallel or perpendicular to the polarization PA. HL Tau and MWC 297, the two YSOs with discrepancies between the jet and disk PA, are more closely correlated with the disk PA. In summary, seven objects present optically thick disks and five optically thin disks. This is shown with the respective label in Table 5. Our classification is also indicated in the histogram of $\Delta_{\text {pol/disk }}$ (Fig. 2). Individual comments for each analysed object are presented in Sect. 5.

It is interesting to note that the optically thick disks of our sample exhibit the highest observed polarizations (Fig. 3). This result is consistent with the expected enhanced polarization by multiple scattering in an optically thick environment (Ménard \& Bastien 1992). Alternatively, these polarization levels can also be explained by less dilution of the observed radiation by direct 
Table 5. Correlations with position angles of jets and disks.

\begin{tabular}{|c|c|c|c|c|c|c|}
\hline Object & $\begin{array}{r}\mathrm{PA}_{\text {jet }} \\
\left({ }^{\circ}\right)\end{array}$ & $\begin{array}{l}\mathrm{PA}_{\text {disk }} \\
\left({ }^{\circ}\right)\end{array}$ & $\begin{array}{l}\Delta_{\text {jet } / \text { disk }}{ }^{a} \\
\left(^{\circ}\right)\end{array}$ & $\begin{array}{l}\Delta_{\text {pol/disk }}{ }^{b} \\
\left(^{\circ}\right)\end{array}$ & $\begin{array}{l}\text { Disk opt. } \\
\text { depth }\end{array}$ & Ref. $^{c}$ \\
\hline \multicolumn{7}{|c|}{ T Tauri } \\
\hline AS 353A & 107 & - & - & 83 & thin & 1 \\
\hline V536 Aql & 110 & - & - & 5 & thick & 2 \\
\hline RY Tau & 115 & $27(7)$ & 2 & 85,87 & thin & 3,4 \\
\hline DG Tau & 42 & $\sim 136$ & 4 & 5,9 & thick & 5,6 \\
\hline DO Tau & 70 & $160(9)$ & 0 & 6,6 & thick & 7,8 \\
\hline HL Tau & 51 & $125(10)$ & 16 & 17,1 & thick & 9,10 \\
\hline $\mathrm{LkH} \alpha 358$ & 72 & - & - & 11 & thick & 11 \\
\hline UY Aur & - & $\sim 135$ & - & 78 & thin & 12 \\
\hline \multicolumn{7}{|c|}{ Herbig $\mathrm{Ae} / \mathrm{Be}$} \\
\hline Hen 3-1191 & 48 & - & - & 87 & thin & 13 \\
\hline PDS 453 & - & $\sim 45$ & - & 13 & thick & 14 \\
\hline MWC 297 & 164 & $\sim 100$ & 26 & 40,14 & thick & 15,16 \\
\hline VV Ser & - & $15(5)$ & - & 83 & thin & 17 \\
\hline
\end{tabular}

${ }^{a} \Delta_{\text {jet } / \text { disk }}=\left|\mathrm{PA}_{\text {jet }}-\mathrm{PA}_{\text {disk }}+180 \times n-90\right|$.

${ }^{b} \Delta_{\mathrm{pol} / \mathrm{disk}}=\left|\mathrm{PA}_{\mathrm{pol}}-\mathrm{PA}_{\text {axis }}+180 \times n-90\right|$ when $\mathrm{PA}_{\text {axis }}=\mathrm{PA}_{\mathrm{jet}}$; or $\Delta_{\mathrm{pol} / \mathrm{disk}}=90-\Delta_{\mathrm{pol} / \mathrm{disk}}$ when $\mathrm{PA}_{\mathrm{axis}}=\mathrm{PA}_{\text {disk }} ; \mathrm{PA}_{\mathrm{pol}}=\mathrm{PA}_{\text {int }}($ when available $)$ or PA obs.

${ }^{c}$ References for $\mathrm{PA}_{\text {jet }}$ and/or PA disk: (1) Curiel et al. (1997); (2) Mundt \& Eislöffel (1998); (3) St-Onge \& Bastien (2008); (4) Kitamura et al. (2002); (5) Lavalley et al. (1997); (6) Testi et al. (2002); (7) Hirt et al. (1994); (8) Koerner \& Sargent (1995); (9) Mundt et al. (1990); (10) Wilner et al. (1996); (11) Moriarty-Schieven et al. (2006); (12) Potter et al. (2000); (13) Le Bertre et al. (1989); (14) Perrin (2006); (15) Drew et al. (1997); (16) Monnier et al. (2006); (17) Pontoppidan et al. (2007).

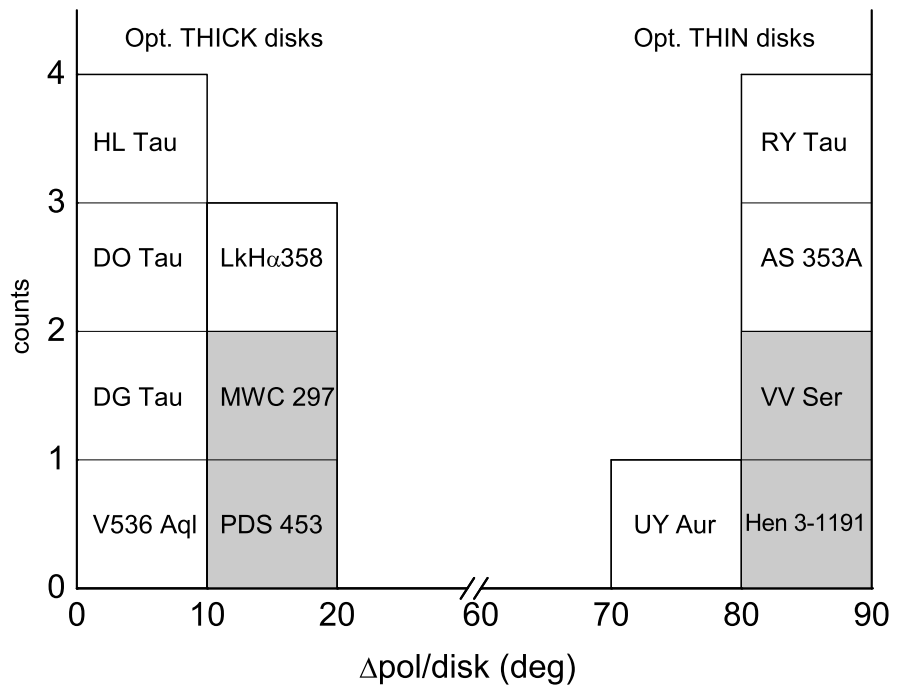

Fig. 2. Histogram of the $\Delta_{\mathrm{pol} / \text { disk }}$ parameter used to classify the disk optical depth at NIR bands. The TTS are in white boxes and the HeAeBeS in grey boxes.

light from the central object in an optically thick line-of-sight (as in UXOR stars - see next section).

\subsection{Comparing optical and NIR polarization PA - cases of possible reversal?}

A change in the polarization PA by $90^{\circ}$ between the visible and the NIR (or polarization reversal) was observed in a couple of YSOs (T Tau and SU Aur, Hough et al. 1981). This feature can be explained by an optically thick disk in the optical bands, which produces a polarization parallel to the disk, that is instead optically thin at NIR bands, resulting in a polarization PA perpendicular to the disk (Bastien \& Ménard 1990).

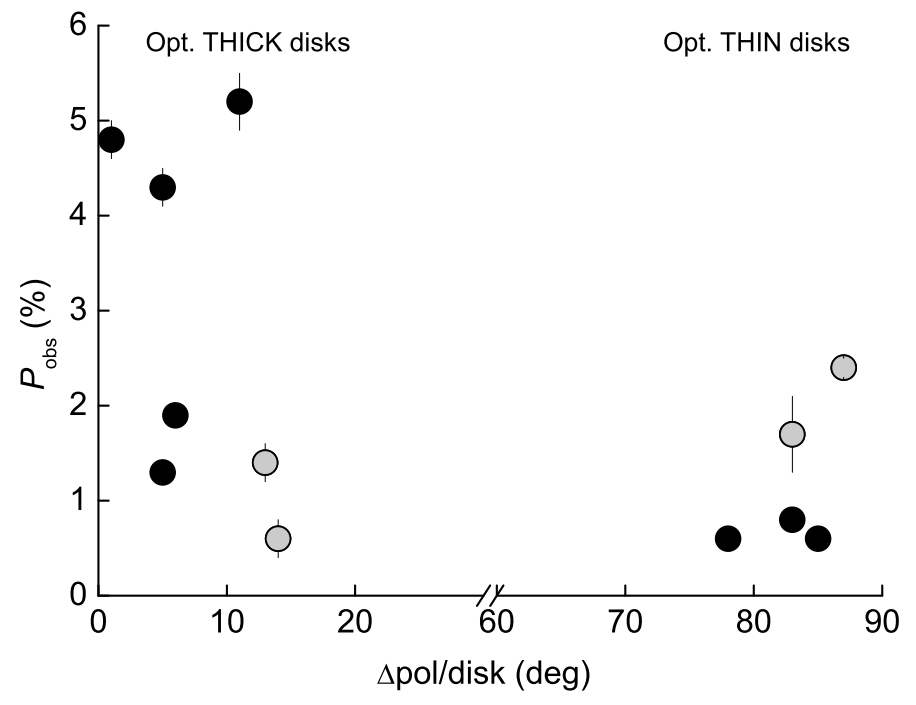

Fig. 3. Correlation between the observed polarization at NIR bands and the $\Delta_{\mathrm{pol} / \mathrm{disk}}$ parameter. The TTS are in black dots and the HeAeBeS in grey dots.

Evidence of polarization reversal in our sample can be obtained by comparing the optical and NIR polarization PA from Tables 3 and 4. A direct comparison can be achieved by analyzing the $Q-U$ diagrams shown in Fig. 1. The polarization reversal is evident when a flip between two opposite quadrants in the $Q-U$ diagram exists between the optical and NIR polarization. Since the comparison involves data for different epochs, our conclusions can be biased by a long-term intrinsic-polarization variability and must be interpreted with care.

Nevertheless, to within $25^{\circ}$ in four objects (RY Tau, UY Aur, RY Ori, and WW Vul) of our sample, polarization reversal seems to be present. The evidence of optically thin disks at NIR bands for RY Tau and UY Aur given in Sect. 4.1 seems consistent with 

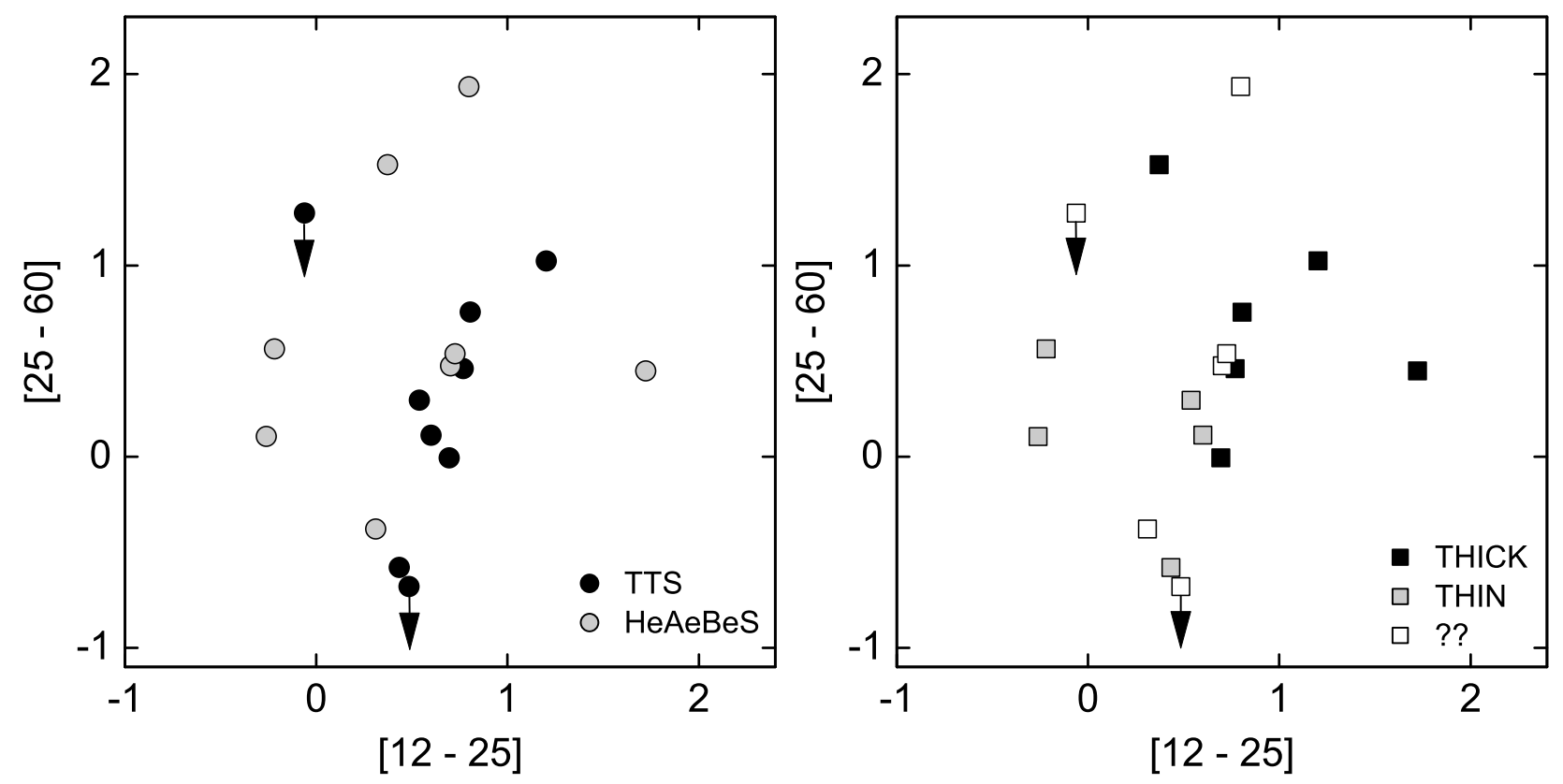

Fig. 4. IRAS color diagram for our sample. (Left) The locus for the TTS (black dots) and the HeAeBeS (grey dots) is shown. (Right) The optically thick disks (black squares) in our sample seems to ocuppy a different locus than the optically thin disks (grey squares). Objects with an undetermined disk optical depth also are shown (white squares).

the polarization-reversal model mentioned above. The indeterminacy of the disk optical depth of RY Ori and WW Vul prevents any conclusion about these two objects.

In particular, the $Q-U$ diagram for RY Tau exhibits significant variability that has already been reported at optical and NIR bands. Our NIR polarization data obtained in 2005 Oct. show a polarization reversal with respect to the temporal evolution in the optical polarization (between 1972 Jan. and 2003 Dec.). On the other hand, previous NIR polarization data over an interval of $\sim 2.5 \mathrm{yr}$ (between 1979 Aug. and $1981 \mathrm{Dec}$.) occupied the same quadrant $(Q>0, U>0)$ as the historical optical variability. UY Aur was reported with a suspected optical polarization variability (Bastien 1985). This object shows a polarization reversal if we compare the optical polarization on 1978 Dec. 1 with our NIR polarization. However, the feature is not evident if we instead compare with the optical data between 1979 Feb. and 1980 Jan. In RY Ori, the detection of the polarization reversal is marginal. The feature is evident only between the first of the two optical data on 1995 Jan. 30 and our NIR data. Finally, in WW Vul, polarization reversal is evident between the optical data on 1998 May and our NIR data but fails if the comparison is made with the optical data on 1998 Jul.-Oct. and 2000 Jun.

It is interesting to note that RY Tau, RY Ori, and WW Vul, which show evidence of a polarization reversal, also exhibit the UXOR phenomenon (Grinin 1994; Yudin \& Evans 1998; Petrov et al. 1999; Oudmaijer et al. 2001). Nevertheless, VY Mon, which also displays the UXOR behavior (Oudmaijer et al. 2001), does not show the polarization reversal in our comparison (Fig. 1). The UXOR phenomenon is associated with an increase in polarization simultaneously to an abrupt decrease in the optical brightness of the central object (Grinin et al. 1994) in nonperiodic variables with deep Algol-type minima. This feature is explained by the effect of dust clumps rotating around the central star in a disk-like configuration. Clumps along our line of sight will absorb the light of the central star increasing the proportion of scattered light and consequently the polarization. When the line of sight is free of clumps, the star will become bright again and the contribution of the scattered light will be reduced along with the polarization. In the optical bands, polarization PA flips are common in stars with the UXOR phenomenon. For example, UX Ori (the prototype for the UXOR phenomenon) shows after the minimum a rapid increase in the polarization level followed by a gradual polarization PA flip of $\sim 90^{\circ}$ (Grinin 1994). A similar behavior was also reported for WW Vul with a polarization PA flip of $\sim 60^{\circ}$ (Grinin et al. 1988) and more recently for CO Ori (Rostopchina et al. 2007, PA flip $\sim 90^{\circ}$ ).

In RY Tau, the historical NIR polarization PA variability would imply significant changes between an optically thick disk, between 1979 Aug. and 1981 Dec. (Hough et al. 1981; Moneti et al. 1984), and an optically thin disk in our NIR data. These changes are probably associated with the screening effect by clumps following the UXOR behavior.

\subsection{IRAS colors and spectral index}

To determine whether any correlations exist between the disk optical depth computed in Sect. 4.1 and the IRAS colors, we plotted in Fig. 4 the IRAS color diagram for our sample. The far-IR colors were calculated using $\left[\lambda_{i}-\lambda_{j}\right]=2.5 \log \left[F\left(\lambda_{j}\right) / F\left(\lambda_{i}\right)\right]$, where $F(\lambda)$ are the IRAS fluxes (Table 6). Only V895 Sco and LkH $\alpha$ 358 have no IRAS counterpart. The HeAeBeS and TTs cover an approximately homogeneous range of the diagram (Fig. 4, left). However, when the positions in the diagram are classified by the disk optical depth, a segregation between the two subsamples becomes evident (Fig. 4, right). The optically thick disks exhibit more far-IR emission than the optically thin ones and an evolutionary segregation is evident. For completeness, we also plotted the objects with undetermined disk optical depth. If the segregation is true, the objects with more far-IR emission are expected to have optically thick disks.

Another way of checking this last result is by computing the spectral index of objects in our sample with a well determined disk optical depth (Fig. 5). We used the classification of Lada (1987) with $\alpha(K, 25)=\log \left[\lambda_{25} F_{K} /\left(\lambda_{K} F_{25}\right)\right] / \log \left(\lambda_{K} / \lambda_{25}\right)$ 


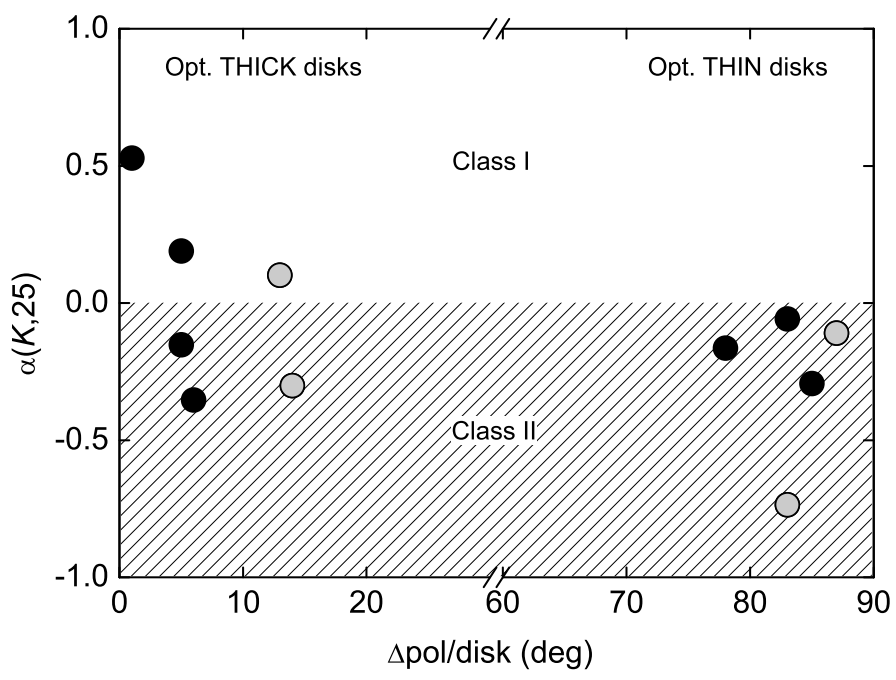

Fig. 5. Spectral index $\alpha(K, 25)$ for the YSOs in our sample with $\Delta_{\mathrm{pol} / \text { disk }}$ parameter determined. Class I and II sources are equally distributed in optically thick disks. Nevertheless, the optically thin disks are only associated to class II sources. The TTS are in black dots and the HeAeBeS in grey dots.

Table 6. Additional information.

\begin{tabular}{llrrr}
\hline \hline Object & IRAS name & {$[12-25]$} & {$[25-60]^{a}$} & $\alpha(K, 25)$ \\
\hline \multicolumn{5}{c}{ T Tauri } \\
AS 353A & $19181+1056$ & 0.54 & 0.30 & -0.06 \\
PX Vul & $19245+2347$ & 0.49 & -0.68 & -0.74 \\
V536 Aql & $19365+1023$ & 0.69 & -0.01 & -0.15 \\
RY Tau & $04188+2819$ & 0.43 & -0.58 & -0.29 \\
DG Tau & $04240+2559$ & 0.81 & 0.76 & 0.19 \\
DO Tau & $04353+2604$ & 0.77 & 0.46 & -0.35 \\
HL Tau & $04287+1807$ & 1.20 & 1.02 & 0.53 \\
UY Aur & $04486+3042$ & 0.60 & 0.11 & -0.16 \\
RY Ori & $05296-0251$ & -0.06 & 1.27 & -0.68 \\
\hline \multicolumn{5}{c}{ Herbig Ae/Be } \\
PDS 406 & $16017-3936$ & 0.80 & 1.94 & 0.26 \\
Hen 3-1191 & $16235-4832$ & -0.26 & 0.11 & -0.11 \\
PDS 453 & $17178-2600$ & 1.72 & 0.45 & 0.10 \\
MWC 297 & $18250-0351$ & 0.37 & 1.53 & -0.30 \\
VV Ser & $18262+0006$ & -0.22 & 0.56 & -0.74 \\
PDS 520 & $18275+0040$ & 0.70 & 0.47 & -0.29 \\
WW Vul & $19238+2106$ & 0.31 & -0.38 & -0.59 \\
VY Mon & $06283+1028$ & 0.72 & 0.54 & 0.12 \\
\hline
\end{tabular}

${ }^{a}$ Upper limits for PX Vul and RY Ori.

representing the spectral index between the 2MASS $K$ band and the $25 \mu \mathrm{m}$ IRAS flux (see Table 6). For class I sources, we have $0<\alpha \leq 3$, and for class II sources, $-2 \leq \alpha \leq 0$. Clearly, all the optically thin disks are associated with class II sources. On the other hand, the optically thick disks appear to be equally distributed between the two evolutionary classes. The link between the class II objects and the optically thin disks reinforces the idea of an evolutionary segregation for the YSO disks. If this is correct, we have found evidence that the disks in YSOs tend to be optically thin when they are older. This picture is consistent with the final phases of disk evolution in the standard star-formation model (Shu et al. 1987).

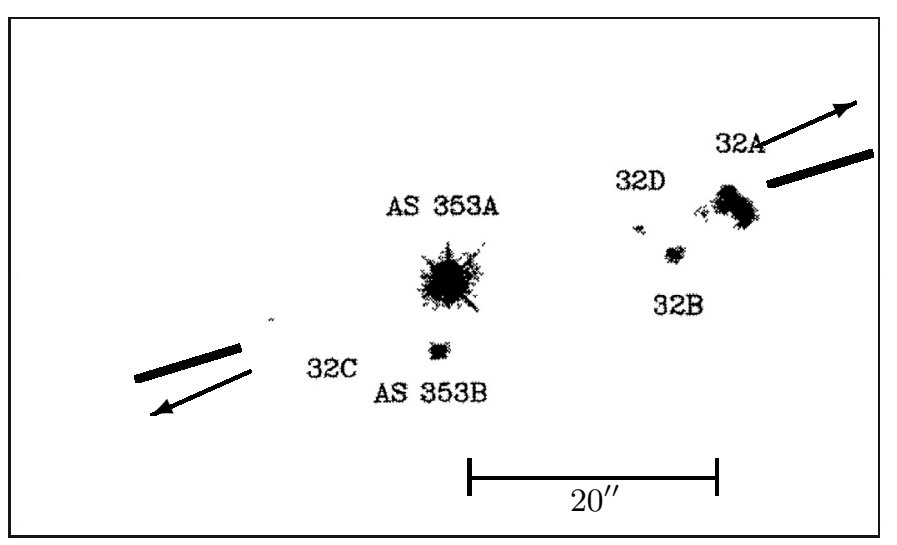

Fig. 6. H $\alpha$ image of AS 353A adapted from Curiel et al. (1997). The $\mathrm{HH}$ objects associated are shown along with the outflow axis defined by them (thick lines). The PA from NIR polarization (corrected by ISP) is also indicated (black arrows). North is top and East is left.

\section{Comments on individual objects}

\section{1. $T$ Tauri stars}

\subsubsection{AS 353A}

AS $353 \mathrm{~A}$ is a TTS member of a binary system with a separation of 5.7 (Reipurth \& Zinnecker 1993). The aperture radius used in our measurement is $1^{\prime \prime} .3$, which avoids contamination by the companion. The outflow axis direction, $\mathrm{PA}=107^{\circ}$ (or $\mathrm{PA}=287^{\circ}$ ), is well determined by the relative positions of its associated HH objects (HH 32A-D, Hartigan et al. 1986) and proper motions (Curiel et al. 1997). Our NIR polarization PA $\left(125^{\circ} \pm 1^{\circ}\right)$ is approximately parallel to the outflow axis. If we consider the ISP correction ( $\mathrm{PA}=114^{\circ} \pm 1^{\circ}$ ), the correlation is even stronger (Fig. 6). This is consistent with the NIR polarization being produced in an optically thin disk.

\subsubsection{V536 Aql}

V536 Aql is a classical TTS. Optical polarimetric variability was reported by Ménard \& Bastien (1992) with a range of polarization $\sim[5.5-8.5] \%$ and PA $\sim[40-70]^{\circ}$ measured during about 9 yrs. Subarcsecond NIR imaging (Ageorges et al. 1994) for V536 Aql detected a binary system with a PA axis $\sim 17^{\circ}$ and an angular distance of $\sim 0.5^{\prime \prime}$. Evidence of the outflow/jet axis was found by Hirth et al. (1997) using long-slit spectra of forbidden emission lines $\left(\mathrm{PA}=90^{\circ} \pm 20^{\circ}\right)$; and by Mundt \& Eislöffel (1998) using [SII]-continuum imaging $\left(\mathrm{PA} \sim 110^{\circ}\right)$. Our measurements do not resolve the system but the NIR polarization PA $\left(25^{\circ} \pm 1^{\circ}\right)$ is approximately parallel to the binary PA axis and also perpendicular to the bipolar outflow (Fig. 7). The NIR polarization probably originates in an optically thick dusty (and circumbinary?) disk.

\subsubsection{RY Tau}

An estimate of the disk PA is found in Kitamura et al. (2002, $\mathrm{PA}=27^{\circ} \pm 7^{\circ}$ ) using $2 \mathrm{~mm}$ thermal dust continuum emission, and Koerner \& Sargent $\left(1995, \mathrm{PA}=48^{\circ} \pm 5^{\circ}\right)$ using $\operatorname{CO}(2 \rightarrow 1)$ emission. Considering the consistent evidence of a disk from $\mathrm{CO}$ and $2 \mathrm{~mm}$ emission, the NIR polarization PA $\left(127^{\circ} \pm 1^{\circ}\right)$ is approximately perpendicular to the disk PA. The correlation becomes even stronger if we consider the correction by ISP $\left(\mathrm{PA}_{\text {int }}=120^{\circ} \pm 00^{\circ}\right.$, Table 4$)$. In addition, using $\mathrm{H} \alpha$-continuum 


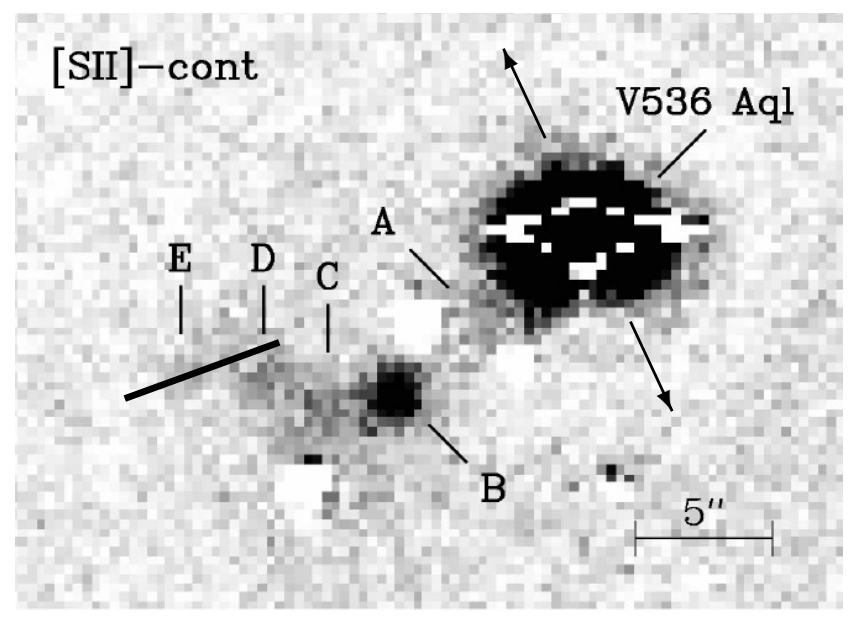

Fig. 7. [SII]-continuum image of V536 Aql adapted from Mundt \& Eislöffel (1998). The jet axis is indicated (thick line) along with the PA from NIR polarization (arrows). North is top and East is left.

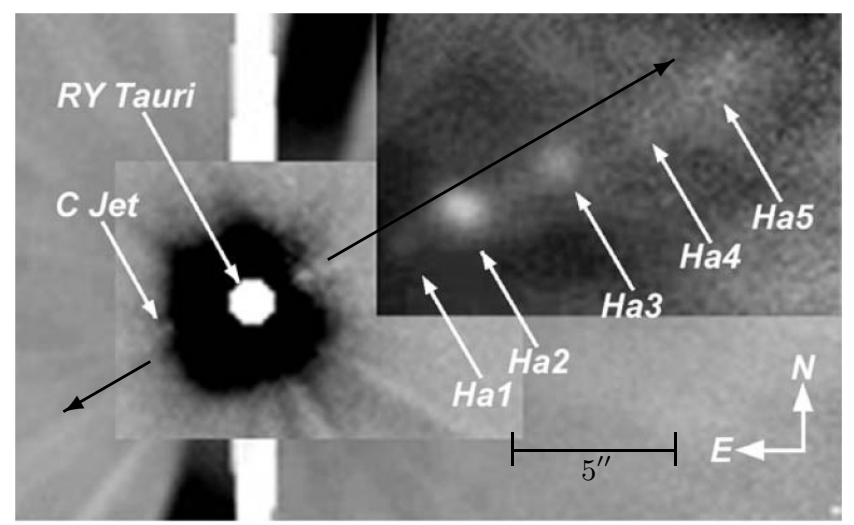

Fig. 8. $\mathrm{H} \alpha$-continuum image of RY Tau adapted from St-Onge \& Bastien (2008). The knots (Ha1 to Ha5) indicate the jet axis. The PA from NIR polarization (corrected by ISP) is also indicated (black arrows).

Gemini imaging, St-Onge \& Bastien (2008) reported a bipolar jet from RY Tau with PA $=115^{\circ}$, approximately parallel to our NIR polarization PA (Fig. 8). This seems to confirm that an optically thin disk is more likely in RY Tau. Nevertheless, previous NIR polarization measurements have reported polarization PAs that differ from our value (see Table 7). This is a clear indication of the significant variability of this source also in the NIR bands. As we noted in Sect. 4.2, the UXOR phenomenon can probably explain the large variations in the polarization PA.

\subsubsection{DG Tau}

The NIR polarization PA $\left(128^{\circ} \pm 2^{\circ}\right)$ is roughly perpendicular to the jet direction $\left(\mathrm{PA}=222^{\circ}\right)$ inferred from $[\mathrm{OI}]$-continuum maps (Lavalley et al. 1997). Evidence of a Keplerian disk rotating perpendicular to the jet was found by Testi et al. (2002) using ${ }^{13} \mathrm{CO}$ wing emission. Therefore, the NIR polarization PA is also parallel to the disk as expected in an optically thick dusty disk (Fig. 9). It is interesting to note that previous published polarization data in $H$ band for DG Tau (see Table 7) detected a higher polarization level but a PA consistent with ours to within $10^{\circ}$. Therefore, the polarimetric variability seems to be unrelated to geometric changes in the material distribution.
Table 7. NIR polarization in $H$ band for RY Tau, DG Tau and, HL Tau from the literature.

\begin{tabular}{rllll}
\hline \hline Object & Date & $P(\%)$ & PA $\left(^{\circ}\right)$ & Ref. \\
\hline RY Tau & 1979 Aug 12 & $2.8(0.1)$ & $12(1)$ & 1 \\
& 1979 Oct 13 & $2.0(0.1)$ & $24(1)$ & 1 \\
& 1979 Nov 12 & $1.2(0.2)$ & $12(2)$ & 1 \\
& 1980 Feb 27 & $1.2(0.1)$ & $41(3)$ & 1 \\
& 1981 Dec 05 & 1.5 & 23 & 2 \\
& 1981 Dec 06 & 1.6 & 9 & 2 \\
& 1981 Dec 07 & 2.1 & 9 & 2 \\
DG Tau & 1981 Dec 05 & $5.1(0.3)$ & 137 & 2 \\
& 1981 Dec 06 & $4.3(0.3)$ & 137 & 2 \\
& 1981 Dec 07 & $4.1(0.3)$ & 138 & 2 \\
HL Tau & 1982 Oct 04 & $3.7(0.3)$ & 137 & 2 \\
\hline
\end{tabular}

Errors in parenthesis.

References: (1) Hough et al. (1981); (2) Moneti et al. (1984); (3) Lucas et al. (2004) with aperture radius of $0.2^{\prime \prime}$.

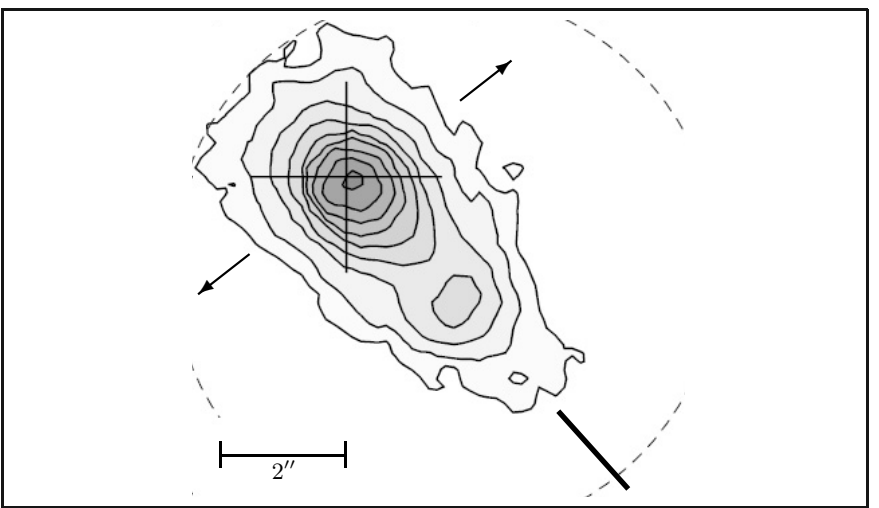

Fig. 9. [OI]-continuum image of DG Tau adapted from Lavalley et al. (1997). The jet axis is indicated (thick line) along with the PA from NIR polarization (arrows). North is top and East is left.

\subsubsection{DO Tau}

The NIR polarization PA $\left(166^{\circ} \pm 1^{\circ}\right)$ is consistent with the optical value $\left(\mathrm{PA}=170^{\circ}\right.$, Bastien 1982). Using [SII] long-slit spectroscopy, Hirth et al. (1994) detected the optical jet direction $\left(\mathrm{PA}=70^{\circ}\right)$. Koerner \& Sargent (1995) determined the disk $\mathrm{PA}\left(160^{\circ} \pm 9^{\circ}\right)$ from $\mathrm{CO}(2 \rightarrow 1)$ emission. The optical jet and the $\mathrm{CO}$ emission seem consistent with the NIR polarization PA being produced in an optically thick dusty disk (Fig. 10). However, Kitamura et al. (2002) estimated a different (and perpendicular) disk PA $\left(67^{\circ} \pm 9^{\circ}\right)$ from dust thermal emission at $2 \mathrm{~mm}$. This discrepancy can probably be explained by the poorly resolved dust emission. The difference between the longer and shorter disk axes, in that work, is lower than $20 \%$.

\subsubsection{HL Tau}

HL Tau is a well-studied T Tauri star. Mundt et al. (1990) determined the jet direction $\left(\mathrm{PA}=51^{\circ}\right)$ using $[\mathrm{SII}]$-continuum imaging. Using different techniques, several works determined a consistent disk PA around of $125^{\circ}$ (Lay et al. 1994; Mundy et al. 1996; Wilner et al. 1996; Close et al. 1997; Murakawa et al. 2008). Different disk PAs were found in Sargent \& Beckwith $\left(1991\right.$, PA $\left.=160^{\circ}\right)$, Stapelfeldt et al. $\left(1995\right.$, PA $\left.=145^{\circ}\right)$, 


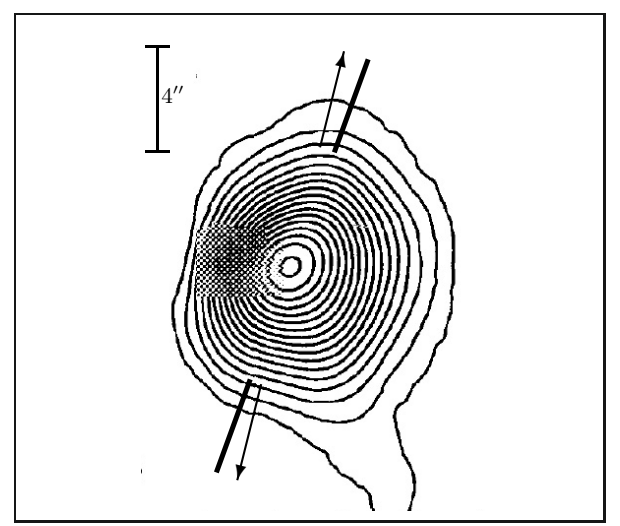

Fig. 10. CO emission map for DO Tau adapted from Korner \& Sargent (1995). The disk PA from CO is shown (thick line) along with the PA from NIR polarization (arrows). North is top and East is left.

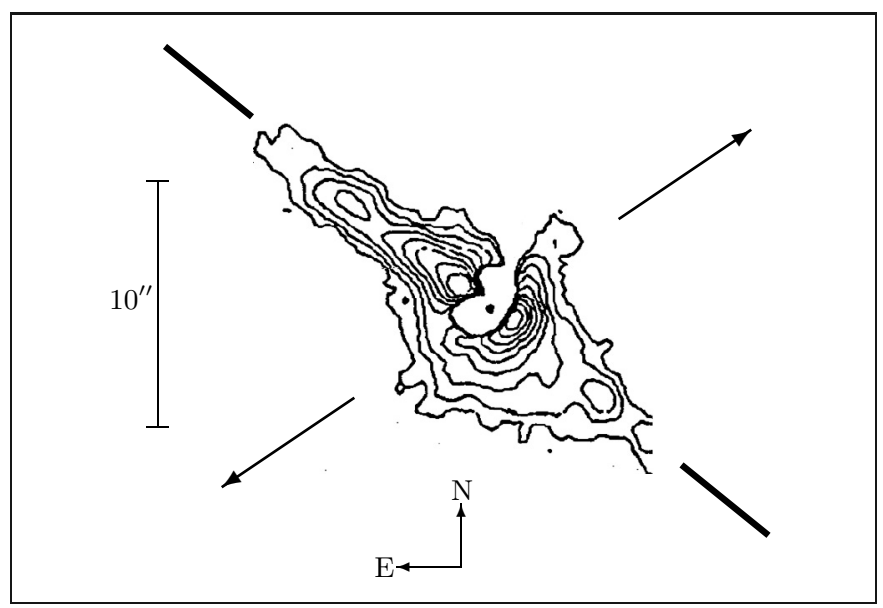

Fig. 11. [SII] $r_{\mathrm{N}}$ image of HL Tau adapted from Mundt et al. (1990) indicating the jets associated (thick line). The PA from NIR polarization is also indicated (arrows).

Kitamura et al. $\left(2002, \mathrm{PA}=144^{\circ} \pm 2^{\circ}\right)$, Lucas et al. $(2004$, $\left.\mathrm{PA}=136^{\circ} \pm 8^{\circ}\right)$, and Rodmann et al. $\left(2006, \mathrm{PA}=170^{\circ} \pm 30^{\circ}\right)$. The evidence of a jet and disk, along with our NIR polarization PA $\left(124^{\circ} \pm 1^{\circ}\right)$, is consistent with an optically thick dusty disk in HL Tau (Fig. 11).

\subsection{7. $\mathrm{LkH} \alpha 358$}

Moriarty-Schieven et al. (2006) suggested that LkH $\alpha 358$ has a jet with PA $=72^{\circ}$. This jet direction is almost precisely perpendicular to the NIR polarization PA $\left(152^{\circ} \pm 2^{\circ}\right)$. Therefore, this is consistent with an optically thick disk in $\mathrm{LkH} \alpha 358$ (Fig. 12).

\subsubsection{UY Aur}

UY Aur is a binary system with a separation of $\sim 0$.' 9 and a binary axis with $\mathrm{PA}=225^{\circ}$ (Leinert et al. 1993). NIR polarization maps of high angular resolution (Potter et al. 2000) inferred a PA for the circumbinary disk of $135^{\circ}$. The large circumbinary disk begins $\sim 2^{\prime \prime}$ from the center of the binary system and continues out to $\sim 5^{\prime \prime}$. We selected a radius aperture of $6^{\prime \prime}$ to properly cover the circumbinary disk. Our NIR polarization PA is $33^{\circ} \pm 5^{\circ}$ approximately perpendicular to the disk PA. It seems consistent with a probably optically thin circumbinary disk in UY Aur.

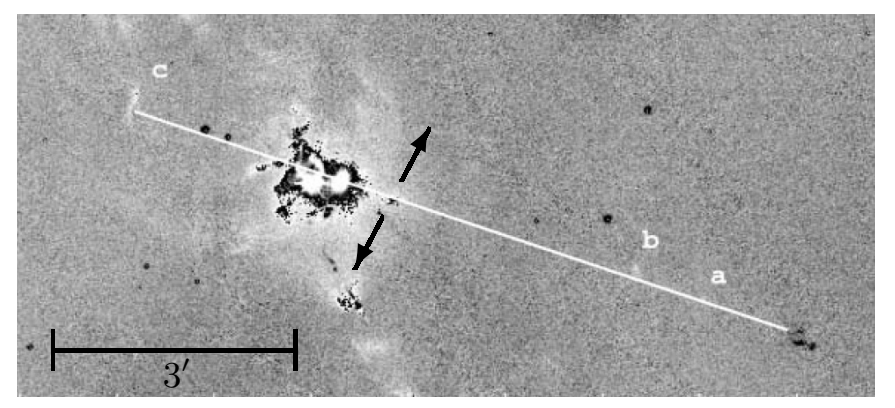

Fig. 12. Jet axis and knots associated (a, b and c) for $\mathrm{LkH} \alpha 358$ adapted from Moriarty-Schieven et al. (2006, white line). The PA from NIR polarization (arrows) is also indicated. North is top and East is left.

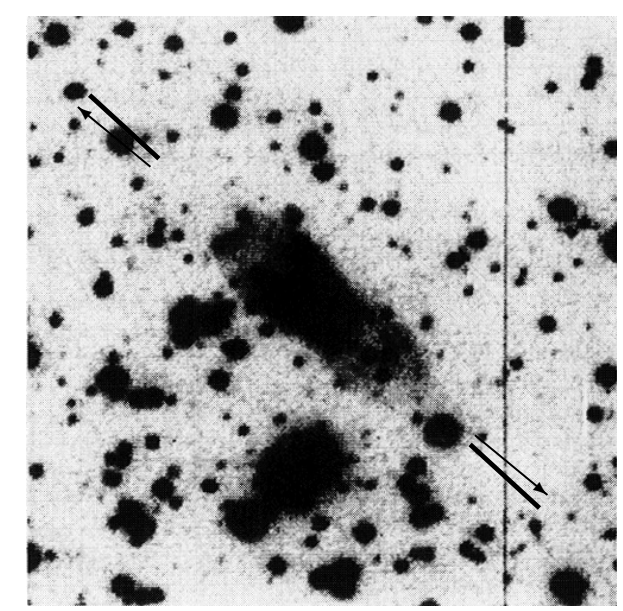

Fig. 13. $R$ frame of Hen 3-1191 from Le Bertre et al. (1989) showing the bipolar structure (axis in thick line). The field is $2^{\prime} \times 2^{\prime}$. The PA from NIR polarization is shown (arrows). North is top and East is left.

\subsection{Herbig Ae/Be stars}

\subsubsection{PDS 406}

PDS 406 is a Herbig Ae star, still embedded in its parental cloud, as suggested by the significant visual extinction $\left(A_{\mathrm{V}}=1\right.$, Chen et al. 1997) and the high NIR foreground polarization $(0.7 \%$, Table 4) toward this object. As we noted in Sect. 3.3, PDS 406 is interesting because it is the object in our sample with the highest $P_{\text {int }}(4.2 \%)$. Viera et al. (2003) detected an $\mathrm{H} \alpha$ double peak that may indicate a rotating disk. Low-resolution (30") maps from ${ }^{12} \mathrm{CO}(J=2 \rightarrow 1)$ (Tachihara et al. 1996) did not detect molecular outflows in PDS 406. High-resolution measurements are needed for this object.

\subsubsection{Hen 3-1191}

The nature of Hen 3-1191 is unclear. It was classified as a protoplanetary nebula (PPN) by Le Bertre et al. (1989). However, De Winter et al. (1994) concluded instead that Hen 3-1191 is probably a pre-main-sequence star. Imaging in $R$-band (Le Bertre et al. 1989) clearly shows a bipolar structure with an axis oriented NW-SE $\left(\mathrm{PA} \sim 48^{\circ}\right)$. The NIR polarization PA $\left(51^{\circ} \pm 1^{\circ}\right.$, corrected by ISP) is almost parallel to the bipolar axis (Fig. 13). This is consistent with scattering in an optically thin disk in Hen 3-1191. Lachaume et al. (2007) resolved Hen 3-1191 at $N$ band $(8-13 \mu \mathrm{m})$ with VLTI/MIDI and found a disk PA of $1^{\circ} \pm 4^{\circ}$. The disagreement with our measurement may be related to the 


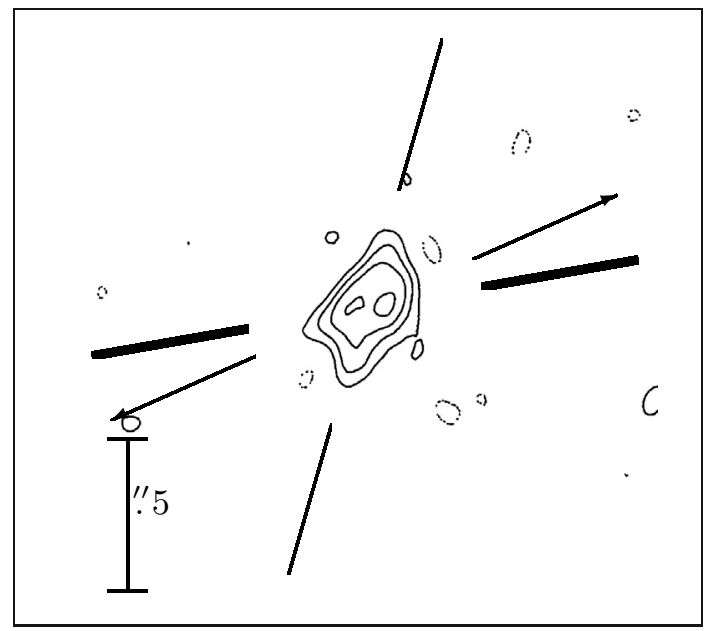

Fig. 14. MERLIN 5Ghz radio map of MWC 297 adapted from Drew et al. (1997). The outflowing axis at $5 \mathrm{Ghz}$ is indicated (thin line). The IOTA interferometer disk PA at NIR from Monnier et al. (2006) also is shown (thick line) along with the PA from NIR polarization corrected by ISP (arrows). North is top and East is left.

high angular resolution (24-36 mas) of the data in Lachaume et al. (2007), which maps the more interior disk region.

\subsubsection{PDS 453}

PDS 453 was classified as a possible PPN by Kohoutek (1997) (see also García-Lario et al. 1997). On the other hand, Vieira et al. (2003) classified PDS 453 as a Herbig Ae/Be candidate but without a clear association with star-forming regions (possibly LDN 1767 and/or 1773). Perrin (2006) at NIR bands detected a possible polarization disk with $\mathrm{PA} \sim 45^{\circ}$ explained by multiple scattering. This PA is approximately parallel to our NIR polarization PA $\left(58^{\circ} \pm 3^{\circ}\right.$, corrected by ISP) and consistent with an optically thick disk.

\subsubsection{MWC 297}

MWC 297 is a Herbig Be star with a well-established spectral type (B1.5Ve, Drew et al. 1997). Using NIR interferometry, Monnier et al. (2006) estimated an elongation of the inner disk with a PA $\sim 100^{\circ}$. This disk PA is approximately parallel to our NIR polarization PA $\left(114^{\circ} \pm 8^{\circ}\right.$, corrected by ISP) and consistent with the NIR polarization produced by multiple scattering (Fig. 14). This seems to confirm previous evidence of an optically thick disk by fitting the SED at $\mathrm{mm} / \mathrm{submm}$ (Mannings 1994) and modeling NIR interferometry data (Malbet et al. 2007). In agreement with this scenario, the radio emission associated with this source $\left(\mathrm{PA}=164^{\circ}\right.$, Drew et al. 1997) is marginally perpendicular to our NIR polarization PA.

\subsubsection{VV Ser}

VV Ser is a Herbig Ae star. Eisner et al. (2004) estimated that it has a disk PA in the $165-173^{\circ}$ range using NIR interferometry. Pontoppidan et al. (2007) studied and modeled Spitzer data of IR continuum and PAH emission for this object with a disk-shadow PA of $15^{\circ} \pm 5^{\circ}$. Our NIR polarization PA $\left(98^{\circ} \pm 7^{\circ}\right.$, corrected by ISP) is approximately perpendicular to the disk PA from Eisner et al. (2004) and Pontoppidan et al. (2007). Therefore, an optically thin disk is more likely in VV Ser (Fig. 15).

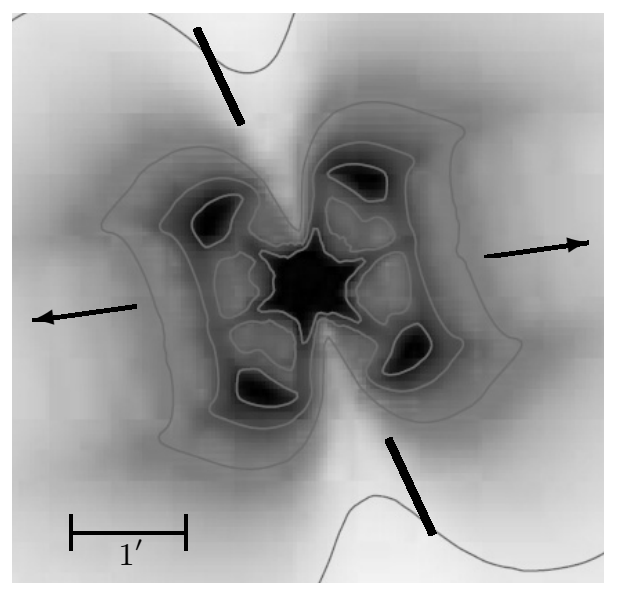

Fig. 15. IRAC $8 \mu \mathrm{m}$ model of VV Ser adapted from Pontoppidan et al. (2007) with the disk PA indicated (thick line). The PA from NIR polarization corrected by ISP is also shown (arrows). North is top and East is left.

\subsubsection{VY Mon}

VY Mon is an eruptive, highly reddened, Algol-like variable Herbig Ae/Be star. VY Mon is associated with the reflection nebula IC 446 (1.5' north) and a Bok globule (1.5' south). There is not clear evidence for a jet/disk in VY Mon. However, evidence of asymmetry was found by modeling the FIR emission (Casey \& Harper 1990). Henning et al. (1998) also found $K$-band and millimeter emissions slightly extended to the south of VY Mon. Our NIR polarization PA $\left(3^{\circ} \pm 2^{\circ}\right)$ is parallel to the axis defined by IC 446 and the Bok globule and also by the millimeter emission. However, it is unclear whether the dust emission (from the Bok globule) and the reflection nebula are tracing the same direction as the circumstellar disk.

\section{Conclusions}

We have presented NIR polarimetric measurements of a sample of YSOs. Except for RY Tau, DG Tau, and HL Tau, the NIR polarization of the remaining sixteen objects is published for the first time.

For eight TTS and four HeAeBeS in our sample, we determined their jet/disk orientation by other means. It enables us to correlate the NIR polarization PA with the disk PA and hence determine the optical depth of the disk. Three TTS present optically thin disks (AS 353A, RY Tau, and UY Aur) and five optically thick disks (V536 Aql, DG Tau, DO Tau HL Tau, and $\mathrm{LkH} \alpha 358$ ). On the other hand, two HeAeBeS show evidence of optically thin disks (Hen 3-1191 and VV Ser) and two of optically thick disks (PDS 453 and MWC 297). Therefore, considering the objects with the correlations available, we found that $\sim 60 \%$ ( 7 of 12 objects) have optically thick disks at NIR bands. Our statistics is limited but our analysis seems to confirm the previous trend of optically thick disks in YSOs (Ménard \& Bastien 1992; Maheswar et al. 2002) obtained at the optical bands.

Marginal evidence of polarization reversal between the optical and NIR bands is found in four objects (RY Tau, UY Aur, RY Ori, and WW Vul). The presence of optically thin disks at the epoch of our observations for RY Tau and UY Aur given by the correlation between the NIR polarization and the jet/disk PA seems consistent with the polarization reversal model. Interestingly, RY Tau, RY Ori, and WW Vul, which show evidence of polarization reversal, exhibit the UXOR behavior. 
In particular, RY Tau shows historical changes in the disk optical depth at NIR bands that we believe are influenced by the presence of eventual dust clumps along the line of sight associated with the UXOR behavior. Probably, RY Ori and WW Vul have similar behaviors but more data are necessary for this to be confirmed.

Finally, we show a correlation between the IRAS colors and the disk optical depth. The optically thick disks are located in a place on the color diagram that corresponds to larger values of far-IR emission. This can be interpreted as an evolutionary segregation. It is corroborated by the fact that only objects classified as Class II sources by their spectral indexes present optically thin disks. Therefore, polarimetric measurements at NIR bands along with independent evidence of the geometry of the disk PA can potentially help to constrain the evolutionary stage of YSOs.

Acknowledgements. The authors wish to thank the anonymous referee for his/her careful reading. His/her several comments and suggestions helped to improve the paper. A.P. thanks FAPESP (grant 02/12880-0) and CNPq (DTI grant $382.585 / 07-03$ associated with the PCI/MCT/ON program). A.P. is also grateful to ALFA/LENAC project for financial support at ICE/Spain. A.M.M. is thankful to FAPESP and CNPq for financial support. J.M.G. is supported by MEC grant AYA2005-08523-C03 (including FEDER funds) and AGAUR grant 2005SGR 00489. Polarimetry at IAG-USP is supported by FAPESP grant $01 / 12589-1$. This research has made use of the VizieR catalogue access tools operated at CDS, Strasbourg, France.

\section{References}

Ageorges, N., Ménard, F., Monin, J.-L., \& Eckart, A. 1994, A\&A, 283, L5 Akeson, R. L., Walker, C. H., \& Wood, K. 2005, ApJ, 622, 440

Angel, J. R. P. 1969, ApJ, 158, 219

Anglada, G. 1996, in Radio Emission from the Stars and the Sun, ed. A. R. Taylor, \& J. M. Paredes, ASP Conf. Ser., 93, 3

Bastien, P. 1982, A\&AS, 48, 153

Bastien, P. 1985, ApJS, 59, 277

Bastien P. 1996, in Polarimetry of the Interstellar Medium, ed. W. G. Roberge, \& D. C. B Whittet, ASP Conf. Ser., 97, 297

Bastien, P., \& Ménard, F. 1990, ApJ, 364, 232

Beckford, A. F., Lucas, P. W., Chrysostomou, A. C., \& Gledhill, T. M. 2008, MNRAS, 384, 907

Breger, M. 1974, ApJ, 188, 53

Brown, J. C., \& McLean, I. S. 1977, A\&A, 57, 141

Casey, S. C., \& Harper, D. A. 1990, ApJ, 362, 663

Chen, H., Grenfell, T. G., Myers, P. C., \& Hughes, J. D. 1997, ApJ, 478, 295

Close, L. M., Roddier, F., Northcott, M. J., et al. 1997, ApJ, 478, 766

Curiel, S., Raga, A. C., Raymond, J. C., Noriega-Crespo, A., \& Cantó, J. 1997, AJ, 114, 2736

De Winter, D., Thé, P. S., \& Pérez, M. R. 1994, in ASP Conf. Ser., 64, 413

Drew, J. E., Busfield, G., \& Hoare, M. G., et al. 1997, MNRAS, 286, 538

Dobashi, K., Uehara, H., Kandori, R., Sakurai, T., \& Kaiden, M. 2005, PASJ, 57, 1

Eisner, J. A., Lane, B. F., Hillenbrand, L. A., Akeson, R. L., \& Sargent A. I. 2004, ApJ, 613, 1049

García-Lario, P., Manchado, A., Pych, W., \& Pottasch, S. R. 1997, A\&AS, 126, 479

Gledhill, T. M., \& Scarrott, S. M. 1989, MNRAS, 236, 139

Grinin, V. P. 1994, in The Nature and Evolutionary Status of Herbig Ae/Be Stars, ed. P. S. The, M. R. Perez, \& E. P. J. Van den Heuvel (San Francisco: ASP), ASP Conf. Ser., 62, 63

Grinin, V. P., Kiselev, N. N., Minikulov, N. Kh., \& Chernova, G. P. 1988, Sov. Astron. Lett., 14, 219

Grinin, V. P., The, P. S., de Winter, D., et al. 1994, A\&A, 292, 165

Hartigan, P., Mundt, R., \& Stocke, J. 1986, AJ, 91, 1357

Henning, Th., Burket, A., Launhardt, R., Leinert, Ch., \& Stecklum, B. 1998, A\&A, 336, 565

Hillenbrand, L. A., Strom, S. E., Vrba, F. J., \& Keene, J. 1992, ApJ, 397, 613

Hirth, G. A., Mundt, R., Solf, J., \& Ray, T. P. 1994, 427, L99

Hirth, G. A., Mundt, R., \& Solf, J. 1997, A\&AS, 126, 437

Hough, J. H., Bailey, J., Cunningham, E. C., McCall, A., \& Axon, D. J. 1981, MNRAS, 195, 429
Kitamura, Y., Momose, M., Yokogawa, S., et al. 2002, ApJ, 581, 357

Koerner, D. W., \& Sargent, A. I. 1995, AJ, 109, 5

Kohoutek, L. 1997, Astron. Nachr., 318, 35

Kusakabe, N., Tamura, M., Kandori, R., et al. 2008, AJ, 136, 621

Lachaume, R., Preibisch, Th., Driebe, Th., \& Weigelt, G. 2007, A\&A, 469, 587

Lada, C. J. 1987, in Star Forming Regions, ed. M. Peimbert, \& J. Jugaku (Dordrecht: Reidel), IAU Symp., 115, 1

Lavalley, C., Cabrit, S., Dougados, C., Ferruit, P., \& Bacon, R. 1997, A\&A, 327, 671

Lay, O. P., Carlstrom, J. E., Hills, R. E., \& Phillips, T. G. 1994, ApJ, 434, L75

Le Bertre, T., Epchtein, N., Gouiffes, C., Heydari-Malayeri, M., \& Perrier, C. 1989, A\&A, 225, 417

Leinert, Ch., Zinnecker, H., Weitzel, N., et al. 1993, A\&A, 278, 129

Lucas, P. W., Fukagawa, M., Tamura, M., et al. 2004, MNRAS, 352, 1347

Magalhães, A. M., Rodrigues, C. V., Margoniner, V. E., Pereyra, A., \& Heathcote, S. 1996, ASP Conf. Ser. 97, ed. W. G. Roberge, \& D. C. B. Whittet, 118

Maheswar, G., Manoj, P., \& Bhatt, H. C. 2002, A\&A, 387, 1003

Malbet, F., Benisty, M., de Wit, W.-J., et al. 2007, A\&A, 464, 43

Mannings, V. 1994, MNRAS, 271, 587

McGregor, P. J., Harrison, T. E., Hough, J. H., \& Bailey, J. A. 1994, MNRAS, 267,775

Ménard, F., \& Bastien, P. 1992, AJ, 103, 564

Monet, D. G., Levine, S. E., Zacharias, N., et al. 2004, BAAS, 205, 4815

Moneti, A., Pipher, J., Helfer, H. L., McMillan, R. S., \& Perry, M. L. 1984, ApJ, 282,508

Monin, J.-L., Ménard, F., \& Peretto, N. 2006, A\&A, 446, 201

Monnier, J. D., Berger, J.-P., Millan-Gabet, R., et al. 2006, ApJ, 647, 444

Moriarty-Schieven, G. H., Johnstone, D., Bally, J., \& Jenness, T. 2006, ApJ, 645, 357

Mundt, R., \& Eislöffel, J. 1998, AJ, 116, 860

Mundt, R., \& Fried, J. W. 1983, ApJ, 274, L83

Mundt, R., Ray, T. P., Bührke, T., Raga, A. C., \& Solf, J. 1990, A\&A, 232, 37

Mundy, L. G., Looney, Leslie, W., Erickson, W., et al. 1996, ApJ, 464, L169

Murakawa, K., Oya, S., Pyo, T.-S., \& Ishii, M. 2008, A\&A, 492, 731

Oudmaijer, R. D., Palacios, J., Eiroa, C., et al. 2001, A\&A, 379, 564

Pereyra, A. 2000, Ph.D. Thesis, Instituto Astronômico e Geofísico, Universidade de São Paulo

Pereyra, A., \& Magalhães, A. M. 2002, ApJS, 141, 469

Perrin, M. D. 2006, Ph.D. Thesis, Univ. of California, Berkeley

Perrin, M. D., Graham, J. R., Kalas, P., et al. 2004, Science, 303, 1345

Petrov, P. P., Zajtseva, G. V., Efimov, Yu. S., et al. 1999, A\&A, 341, 553

Pontoppidan, K. M., Dullemond, C. P., Blake, G. A., et al. 2007, ApJ, 656, 991

Potter, D. E., Close, L. M., \& Roddier, F. 2000, ApJ, 540, 422

Qi, C., Kessler, J. E., Koerner, D. W., Sargent, A. I., \& Blake, G. A. 2003, ApJ, 597, 986

Reipurth, B., \& Zinnecker, H. 1993, A\&A, 278, 81

Rodmann, J., Henning, Th., Chandler, C. J., Mundy, L. G., \& Wilner, D. J. 2006, A\&A, 446, 211

Rodrigues, C. V., Sartori, M. J., Gregorio-Hetem, J., \& Magalhães, A. M. 2009, ApJ, accepted, [arXiv: astro-ph/09044812]

Rostopchina, A., N., Grinin, V. P., Shakhovskoi, D. N., et al. 2007, AZh, 84, 60

Sargent, A. I., \& Beckwith, S. V. W. 1991, ApJ, 382, L31

Serkowski, K., Mathewson, D. L., \& Ford, V. L. 1975, ApJ, 196, 261

Schulte-Ladbeck, R. 1983, A\&A, 120, 203

Shu, F. H., Adams, F. C., \& Lizano, S. 1987, ARA\&A, 25, 23

Stapelfeldt, K. R., Burrows, C. J., Krist, L. E., et al. 1995, ApJ, 449, 888

St-Onge, G., \& Bastien, P. 2008, ApJ, 674, 1032

Tamura, M., \& Sato, S. 1989, ApJ, 98, 1368

Tachihara, K., Dobashi, K., Mizuno, A., Ogawa, H., \& Fukui, Y. 1996, PASJ, 48, 489

Testi, L., Bacciotti, F., Sargent, A. I., Ray, T. P., \& Eislöffel, J. 2002, A\&A, 394, L31

Vieira, S. L. A., Corradi, W. J. B., Alencar, S. H. P., et al. 2003, AJ, 126, 2971

Vink, J. S., Drew, J. E., Harries, T. J., Oudmaijer, R. D., \& Unruh Y. 2005, MNRAS, 359, 1049

Vrba, F. J., Schmidt, G. D., \& Hintzen, P. M. 1979, ApJ, 227, 185

Wang, S., \& Looney, L. W. 2007, ApJ, 659, 1360

Whitney, B. A., \& Hartmann, L. 1992, ApJ, 395, 529

Whitney, B. A., \& Hartmann, L. 1993, ApJ, 402, 605

Whitney, B. A., Kenyon, S. J., \& Gómez, M. 1997, ApJ, 485, 703

Whittet, D. C. B., Martin, P. G., Hough, J. H., et al. 1992, ApJ, 386, 562

Wilner, D. J., Ho, P. T. P., \& Rodríguez, L. F. 1996, ApJ, 470, L117

Yudin, R. V., \& Evans, A. 1998, A\&AS, 131, 401 